\title{
Molecular Dynamics Simulations and Thermal Transport at the Nano-Scale
}

\author{
Konstantinos Termentzidis ${ }^{1,2}$ and Samy Merabia ${ }^{3}$ \\ ${ }^{1}$ CETHIL-UMR5008, INSA de Lyon, CNRS, Université Lyon 1, \\ ${ }^{2}$ EM2C-UPR288, Ecole Centrale Paris, CNRS, \\ ${ }^{3}$ LPMCN-UMR5586, Université Lyon 1, \\ France
}

\section{Introduction}

This chapter presents an overview of the Molecular Dynamics (MD) simulation technique to predict thermal transport properties of nanostructured materials. This covers systems having characteristic lengths of the order of a few nanometers like carbon nanotubes, nanowires and also superlattices, i.e. composite materials made of submicronic thickness of solid layers. The common features of these systems is the small ratio between their characteristic system size and the phonon mean free path, which leads to ballistic heat transport and deviations from the classical Fourier law. Also when the density of interfaces gets large, the energy transport properties of the materials can not longer be described solely by the thermal conductivities of the constituents of the material, but depend also on the thermal boundary resistance which measures the transmission of phonons across an interface. In this context, molecular dynamics was proven to be a very useful technique to study heat transport in nanostructured materials. The main reasons are; the length scale probed by the method is in the nanometer range, and it does not make any assumption on the phonons dynamics except their classical nature.

In this contribution, we present two MD methods, the equilibrium and the non-equilibrium method, which are now commonly used to determine both the thermal conductivity and the thermal boundary resistance of nanostructured materials. We focus on superlattices and discuss how the structural features of the interfaces like height, shape, inter-diffusion phenomena and the layer thickness affect the thermal conductivity of the superlattice. We show how these complex phenomena can be predicted by simple models of Lennard-Jones crystals with a mass ratio corresponding to the acoustic impedance ratio of $\mathrm{Si} / \mathrm{Ge}$ and GaAs/AlAs superlattices.

\section{Molecular dynamics}

The development of molecular simulations began in the early fifties after the considerable development of computer facilities in the United States during World War II. A few years after the first Monte-Carlo simulation, Alder and Wainwright, first introduced in the late 50's the Molecular Dynamics (MD) method (Alder \& Wainwright, 1957, 1959). The aim of 
the first simulations both Monte-Carlo or MD was to probe the different phases of model hard spheres. The necessity to model liquids motivated the development of realistic potentials. Rahman was the first to model Argon using Lennard-Jones potential, which is still considered as a standard potential for MD (Rahman, 1964). This opened the way to consider a broad range of condensed matter systems, ranging from liquid water first modelled by Rahman and Stillinger to silicon (Rahman \& Stillinger, 1974). The efforts towards realistic modelling have permitted to applicate MD to characterize the collective excitations in solids (Hensen 1976). The last step came with the implementation of MD thermostats opening the way to probe the phonon dynamics in rare-gas solids (Ladd 1986). More recently, there has been an increasing number of MD studies on nano-scale heat transport motivated by fine measurements of energy transport in nano-materials (Volz 1999). We refer the reader to the classical textbooks on MD (Allain \& Tildesley 1987 and Frenkel \& Smit 1996) for a thorough introduction.

MD is a simulation method based on the numerical integration of Newton's equation of motion:

$$
m \frac{d^{2} \vec{r}_{i}}{d t^{2}}=-\frac{\partial V}{\partial \vec{r}_{i}}
$$

where: $m, \vec{r}_{i}$ and $V$ denote respectively the mass of the particle i, its position vector and the inter-atomic potential. The integration scheme commonly used is the Verlet algorithm (Verlet 1967a, 1967b) which predicts the positions at time $t+d t$ given the positions at the earlier times $t$ and $t-d t$ :

$$
\vec{r}_{i}(t+d t)=2 \vec{r}_{i}(t)-\vec{r}_{i}(t-d t)+\frac{d^{2} \vec{r}_{i}}{d t^{2}} d t^{2}+O\left(d t^{4}\right)
$$

where the acceleration is calculated using the inter-atomic forces. The velocities can be computed using

$$
\vec{v}_{i}(t)=\frac{\vec{r}_{i}(t+d t)-\vec{r}_{i}(t-d t)}{2 d t}+O\left(d t^{2}\right)
$$

and the error is larger than the error on the positions, but as soon as the velocities are only used to compute the instantaneous kinetic energy the consequences are minor. Of course there are other integration schemes, but the Verlet algorithm has the advantage to be simple, easy to implement, stable and time-reversible. The typical time step used in the integration algorithms is a fraction of the characteristic atomic period

$$
\tau=\sqrt{\frac{m \sigma^{2}}{k_{B} T}} \approx 1 p s
$$

where $m$ is the mass of the particles and $\sigma$ denotes a typical atomic diameter.

Given the power of modern computers, it takes a few hours on a mono-processor machine to follow the trajectory of a set of 10000 particles over a time comparable to $1 \mathrm{~ns}$. Using parallel MD codes or GPUs (graphics processing units) opens the way to model larger 
systems or reach orders of magnitude longer times. The system represented in a MD simulation has microscopic dimensions, and usually one is not so much interested in simulating a nano system with free surfaces, but rather a part of a bulk system. To embed the small system simulated in a bulk-like system, one can use periodic boundary conditions. These conditions assume that the system is repeated periodically in all space directions and thus any particle close to the boundaries of the simulation box interacts with an image particle, and when a particle crosses one of the face of the central simulation box, one of its images will enter the central box through the opposite face. One of the consequences of the use of periodic boundary conditions is the cut-off of long wavelengths fluctuations, i.e. those having a wavelength larger than the simulation box length. In the context of heat transport simulations this will imply that phonons with long wavelengths are not present.

The strength of MD lies on the versatility and the flexibility in the choie of the atomic potential (eq.1). Generally speaking, the total potential can be decomposed in the sum

$$
V\left(\vec{r}_{i}\right)=\sum_{i} V_{1}\left(\vec{r}_{i}\right)+\sum_{i<j} V_{2}\left(\vec{r}_{i}, \vec{r}_{j}\right)+\sum_{i<j<k} V_{3}\left(\vec{r}_{i}, \vec{r}_{j}, \vec{r}_{k}\right)+\ldots
$$

where the first term represents the one body contribution to the potential and is often associated to an external field like e.g. an electric field. The second and third terms represent respectively two-body and three-body potentials of the inter-atomic interaction. The choice of the inter-atomic potential depends on the problem at hand (type of atomic structure and on the properties to be studied), and on the necessity to model a real system or an experiment. One of the simplest potential used in condensed matter physics is the LennardJones (LJ) potential, where the total potential decomposes in a sum of pair potentials:

$$
V=\sum_{i<j} V_{2}\left(r_{i j}\right)
$$

with

$$
V_{2}\left(\vec{r}_{i j}\right)=4 \varepsilon\left(\left(\sigma / r_{i j}\right)^{12}-\left(\sigma / r_{i j}\right)^{6}\right)
$$

This is the LJ pair potential, which depends only on the distance between neighbouring particles $r_{i j}=\vec{r}_{i j}$. The interaction energy $\varepsilon$ identifies with the depth well of the potential, and $\sigma$ is the atomic diameter. Although very simple, the LJ potential is often thought to be a good potential to model rare gas and in particular Argon, using the following parameters: $\varepsilon=1,67 \times 10^{-21} \mathrm{~J}$ and $\sigma=3,40 \times 10^{-10} \mathrm{~m}$. Due to the algebraic decay of the LJ potential, all the atoms of the simulation box interact. From a practical point of view however, it would be computationally costly to estimate $N^{2} / 2$ inter-atomic forces at each time step, with $N \approx 10000$. To bring back the computational cost of the force calculation to $O(N)$, the interatomic pair potential is often truncated at a cut-off radius, usually $r_{c}=2,5 \sigma$, which represents typically a neighbourhood of 50 atoms per particle. It should be mentioned at this point, that even if the potential at the radius of truncation is small compared to the well depth: $V\left(r_{c}\right) \approx 0,016 \cdot \varepsilon$, truncating the potential may have a non negligible effect on the thermodynamics of the system modelled. As an example, let us estimate the effect of truncating the inter-atomic potential respectively on the internal energy per particle and on the pressure. The respective errors are given by: 


$$
\Delta U=\rho \int_{r_{c}}^{+\infty} 4 \pi r^{2} V_{2}(r) d r \approx 1,07 \rho \sigma^{3} \varepsilon
$$

and

$$
\Delta P=\frac{-\rho^{2}}{6} \int_{r_{c}}^{+\infty} r \frac{\partial V_{2}}{\partial r} 4 \pi r^{2} d r \approx-1.07 \rho^{2} \sigma^{3} \varepsilon
$$

where we have used the previous value of the cut-off radius and we have assumed that beyond $r_{c}$, the medium is structureless, i.e. the pair correlation function may be considered close to unity. The previous errors are found to be typically $10 \%$ the values of the internal energy and pressure for a condensed phase. This is not worrying since the calculation of thermodynamic quantities can be corrected using the previous estimations, but this has to be kept in mind when calculating a phase diagram for instance.

Although the LJ potential is quite simple, some situations require to use more sophisticated semi-empirical potentials whose parameters are chosen to reproduce either microscopic or macroscopic properties of a model system. These semi-empirical potentials are usually no longer pair potentials like the LJ potential but many-body, where the many-body terms describes how does the potential energy of an atom depends on its coordination.

As an illustration, let us mention the embedded atom model (EAM) used in the context of metals (Daw \& Baskes 1984), where the potential

$$
\sum_{i<j} \phi\left(r_{i j}\right)+\sum_{i} u\left(\rho\left(r_{i}\right)\right)
$$

consists of a pair term $\phi(r)$ accounting for repulsion at short distances and a many body term $u(\rho(r))$ which accounts for the cohesion of the metallic bond and which depends on the local density measured using the positions of neighbouring particles $\rho\left(r_{i}\right)=\sum_{j} w\left(r_{i j}\right)$. The choice of the functional $u(\rho)$ and the weight function $w(r)$ depends on the microscopic and macroscopic properties to be reproduced, usually the lattice constant, the elastic constants and the sublimation energy of the metal (Daw \& Baskes 1984).

Another limitation of LJ potentials is the description of solids crystalizing in non-compact structures. Indeed, Lennard Jones atoms form close-packed structures at low temperatures (usually fcc at low pressure), and thus LJ potentials are also not adapted to model materials which crystallize in opened structures such as diamond. Semi-conductors like silicon or germanium are usually modelled using many-body semi-empirical potentials, in which the many body terms account for the local preferential bond ordering of the semi-conductor. Among the most famous potentials for silicon, let us quote the Tersoff potential (Tersoff 1986, 1988a, 1988b) and the Stillinger-Weber potential (Stillinger \& Weber 1985). The parameters of the former are chosen so as to reproduce the elastic properties of bulk silicon, while the latter describes satisfactorily the structural properties of liquid silicon. Of course the list of potentials is non exhaustive and there exists plethora of empirical potentials to model as disparate systems as charged systems, liquid crystals, polymers, surfactants, granular media,... and mixtures of them! 
As we have already briefly mentioned, the output of MD simulations can be compared to experiments if we know how to relate the microscopic state of the material under study to the macroscopic observables typically measured in an experiment. Generally speaking, one can distinguish three classes of information that can be extracted from MD. On the one hand, MD allows to compute global quantities which correspond usually to the thermodynamics of the system modelled. For instance, the internal energy can be expressed as:

$$
U=\left\langle\sum_{i} V\left(r_{i}\right)\right\rangle+K E
$$

and the total kinetic energy:

$$
K E=\left\langle\sum_{i} \frac{1}{2} m \vec{v}_{i}^{2}\right\rangle
$$

where the brackets denote an ensemble average. If sufficient time has been left to the system to reach equilibrium, the ensemble average may be performed by averaging over a sufficient long time thanks to ergodicity. On the other hand, in non-equilibrium situations, different initial conditions (usually the positions and the velocities of the atoms) may be used to generate independent trajectories in phase space. The latter definition of the kinetic energy is intimately related to the definition of the local temperature :

$$
T(\vec{r})=\left\langle\sum_{i \in V(\vec{r})} \frac{m \vec{v}_{i}^{2}}{3 n(\vec{r}) k_{B}}\right\rangle
$$

where the sum runs over particles $i$ in a small volume $V(r)$ centred around $r$ and which contains $n(r)$ particles. Another important quantity in heat transport simulations is the energy flux, defined as

$$
\vec{J}=\frac{d}{d t}\left(\sum_{i} E_{i} \vec{r}_{i}\right)
$$

where $E_{i}$ is the total energy of particle i, yielding for pair potentials:

$$
\vec{J}=\sum_{i} E_{i} \vec{v}_{i}+\frac{1}{2} \sum_{i<j} \vec{F}_{i j} \cdot\left(\vec{v}_{i}+\vec{v}_{j}\right) \vec{r}_{i j}
$$

For simulations in crystals at low temperatures, the previous energy flux displays large oscillations due to optical phonons which carry a negligible amount of heat, and sometimes it can be more suited to work with the equivalent definition of the flux:

$$
\vec{J}=\frac{1}{2} \sum_{i<j} \vec{F}_{i j} \cdot\left(\vec{v}_{i}+\vec{v}_{j}\right) \vec{r}_{i j}^{0}
$$

where the superscript " 0 " denotes the equilibrium positions of the atoms. From the knowledge of the local temperature and the energy flux, it is possible to measure thermal conductivity and we would come back to the measurement of transport coefficients in MD in the next section, with a particular emphasis on heat transport. 
The other type of information that one can extract from MD is structural. Since the output of a MD simulation is the set of positions $\vec{r}_{i j}$, it is possible to compute the pair distribution function

$$
\rho g(\vec{r})=\left\langle\frac{1}{N} \sum_{i \neq j} \delta\left(\vec{r}-\vec{r}_{i j}\right)\right\rangle
$$

where $\rho$ is the mean number density of the system. Physically, $\mathrm{g}(\mathrm{r})$ represents the probability to find two particles separated by a distance $r$, and $4 \pi r^{2} \rho g(r) d r$ is the average number of particles located at a distance between $r$ and $r+d r$ from a given particle. Note that sometimes, it may be more useful to compute the structure factor:

$$
S(\vec{q})=\left\langle\frac{1}{N} \sum_{i, j} \exp \left(i \vec{q} \cdot\left(\vec{r}_{i}-\vec{r}_{j}\right)\right)\right\rangle
$$

which is simply related to the pair distribution function through a Fourier transform:

$$
S(\vec{q})=1+\rho \int g(\vec{r}) \exp (-i \vec{q} \cdot \vec{r}) d \vec{r}
$$

Finally, MD may be used to compute the vibrational properties of a model system. In particular, the vibrational density of states (DOS)

$$
g(\omega)=\frac{1}{V} \sum_{k} \delta\left(\omega-\omega_{k}\right)
$$

where the sum runs over the eigenmodes of the system can be estimated using the Fourier transform of the velocity autocorrelation function:

$$
g(\omega)=\int_{0}^{\infty}\langle\vec{v}(t) \cdot \vec{v}(0)\rangle \exp (-i \omega t) d t
$$

where here $\vec{v}(t)$ is the velocity of an atom. One has to keep in mind that the previous equation is only approximate, as it assumes small deviations from harmonicity, and thus is only valid strictly speaking at low temperatures. Note also that the resulting DOS mixes information about the different polarizations of the atomic vibrations, e.g. transversal or longitudinal, and to determine the DOS relative to a particular polarization, a more refined analysis of the atomic displacement should be undertaken. Apart from the DOS, MD is a common technique to characterise the propagation of phonons in a crystal.

The vibrations of a crystal containing $\mathrm{N}$ atoms may be decomposed in normal modes:

$$
S_{\vec{k}, v}(t)=N^{-1 / 2} \sum_{i} m_{i}^{1 / 2} \exp \left(-i \vec{k} \cdot \vec{r}_{i}^{0}\right) \vec{e}(\vec{k}, v) \cdot \vec{u}_{i}
$$

where $\vec{u}_{i}(t)$ denotes the displacement of atom $\mathrm{i}$ in the crystal vibrating around the equilibrium position $\vec{r}_{i}^{0}, v$ is an index designating the polarization of the vibration, e.g. longitudinal or transversal and $\vec{e}(\vec{k}, v)$ is the corresponding polarization vector. From the normal mode amplitude, one can compute the dispersion relation and the phonon lifetime as follows. First consider the autocorrelation of the atomic displacement: 


$$
\left\langle u S_{\vec{k}, v}(t) \bar{S}_{\vec{k}, v}(0) \ddot{u}\right.
$$

It is a straightforward exercize to show that

$$
\left\langle u ̈ S_{\vec{k}, v}(t=0) \text { ư }\right\rangle=k_{B} T / w_{\vec{k}}^{2}
$$

Hence, from the previous autocorrelation function, one can determine the dispersion relation $\omega=\omega(\vec{k})$ for the two polarizations. From the dispersion relation, the phonon phase $v_{\phi}(\vec{k})=\omega /(\vec{k})$ and group velocities $\vec{v}_{g}(\vec{k})=\partial \omega / \vec{\lambda} \vec{k}$ may be predicted.

Secondlv, from the time decay of $\left\langle\left(S_{\vec{k}, v}(t) \bar{S}_{\vec{k}, v}(0)\right)\right\rangle$, one can compute the lifetime of the mode $\vec{k}, v$ (Ladd 1986):

$$
\tau_{\vec{k}, v}=\frac{\int\left\langle S_{\vec{k}, v}(t) \bar{S}_{\vec{k}, v}(0)\right\rangle d t}{\left\langle\ddot{u} S_{\vec{k}, v}(t=0) \text { ư }\right\rangle}
$$

The wavelength dependance of the phonon lifetime may then be compared to the theoretical predictions, given either by Callaway 1959 or Holland 1963, see e.g. (McGaughey 2004).

As we have already mentioned, the conversion of the microscopic information given by a MD simulation to macroscopic observables requires to evaluate averages over phase space. The phase space is a multidimensional space generated by the positions and the momenta of a classical system, and has dimension of $6 \mathrm{~N}$ for a system of $\mathrm{N}$ particles. A molecular dynamics simulation generates a sequence of points in phase space as a function of time, whose points belong to the same thermodynamic ensemble. The first MD simulations were performed in the micro-canonical ensemble or NVE ensemble, which corresponds to a fixed number of atoms $\mathrm{N}$, a fixed volume $\mathrm{V}$, and a fixed energy $\mathrm{E}$. Although these simulations are very easy to implement, they do not represent a realistic system as the set of molecules studied is completely isolated. The need to model real situations led to the development of thermostats for MD, which allow to simulate the dynamics of a system in the canonical or the grand-canonical ensemble for instance (Frenkel \& Smit, 1996).

Although MD can be used to calculate the thermodynamics, structural and vibrational properties of systems at equilibrium, it is more designed to study the non-equilibrium situations where the system under study is driven by an external force; the latter can be of thermodynamic nature. In the language of out of equilibrium statistical physics, MD can help in determining the relation between the forces and the fluxes. Statistical physics predicts that there is proportionality between the forces and the fluxes if the system under study is driven weakly out of equilibrium. The coefficients of proportionality are called transport coefficients and measure the susceptibility of the system to respond to a given thermodynamic force. For instance, the thermal conductivity quantifies the amount of energy flowing in a material submitted to a temperature gradient. MD is a very powerful tool to calculate the transport coefficients of a model system. To this end, two routes can be traditionally followed: either the model system is submitted to an external force or the thermally induced fluctuations of an internal variable are probed at equilibrium. Statistical physics states indeed that the typical time decay of the spontaneous thermal fluctuations is 
proportional to the appropriate transport coefficient. This will be illustrated in the context of the heat transfer in the next sextion. Before focusing on energy transfer applications of MD, let us mention that MD can be used also beyond the linear response domain of out of equilibrium systems. This includes situations of large external forcing such as e.g. polymer melts flowing at large shear rates (Vladkov 2006b). But this includes also systems for which intrinsically the linear relationship between forces and fluxes is violated. As an example consider a nano-structured system at low temperatures for which the phonon mean free path is comparable with the characteristic system size. Heat transfer in such systems is no longer described by Fourier law, but is rather described by an effective conductivity which depends on the strength of the thermal flux flowing across the system. Molecular dynamics has been shown to predict the effective conductivity under conditions where the heat carriers travel ballistically in the system and being scattered only by the boundaries of the nanostructure.

\subsection{Limitations of molecular dynamics}

As any simulation technique, molecular dynamics suffers from some intrinsic limitations. The most obvious is the limitation in system size. This may be critical in the modeling of real scale devices with disparate length scales ranging from $1 \mathrm{~nm}$ to microns. To address such situations, MD should be coupled with a more mesoscopic method, such as the Boltzmann Transport Equations, where the microscopic information on the phonon lifetime is used as input in a Boltzmann equation.

We have also mentioned that a finite system size cuts the long wavelength phonon modes. As we will see, this affects only mildly the measurement of the thermal conductivity unless we couple the system with heat reservoirs.

The second limitation of MD is its classical nature. Each phonon mode is equally populated, and the heat capacity is given to a good approximation by the Dulong-Petit law. This is a good approximation if one ever considers solids above or just below their Debye temperature. However, many materials do not obey this condition at ambient temperature. Quantum effects in MD may be accounted for in different ways. The phonon lifetimes computed from MD may be used in a Boltzmann transport equation which includes quantum statistics in the phonon occupation number (McGaughey 2004). Quantum effects may be also directly incorporated in the course of a MD simulation, using a Langevin thermostat with a colored noise consistent with a Bose-Einstein distribution for the phonon modes (Dammak2009).

Finally, the electronic degrees of freedom are not explicitly simulated in MD. Hence, it is not possible to probe thermal transport in electrical conductors, and in its basic version the contribution of electron-phonon scattering to the transport in semi-conductors is not accounted for.

\section{Predicting thermal conductivity with EMD and NEMD}

In general the heat energy is transmitted through a solid by electrons (mainly in metals) or phonons (mainly in insulators) or other excitations as spin waves. In this paragraph, we focus on the lattice thermal conductivity, which is the dominant mechanism in 
semiconductors (Yang, 2004). There are three principal techniques used to evaluate the thermal conductivity with molecular dynamic simulations (Allen \& Tildesley 1987, Chantrenne, 2007); a. the equilibrium approach based on the Green-Kubo formulae (Frenkel \& Smit, 1996), b. the non-equilibrium MD, also called the direct method, which uses a heat source and a heat sink, or the so-called direct method, based on the creation of a temperature gradient, and c. the homogeneous non-equilibrium MD, where a heat flux is induced (Evans, 1982, 1986). The comparison between the two methods has been undertaken by many researchers, who have concluded that the two methods give consistent results (Shelling et al, 2002; Mahajan et al, 2007; Termentzidis et al, 2011b).

\subsection{Non-equilibrium molecular dynamics method (NEMD)}

Kotake and Wakuri proposed the direct method (Kotake \& Wakuri, 1994), which is similar to the hot-plate experiment setup. A temperature gradient is imposed across the structure under study by allowing thermal power exchange between the heat source and sink and measure the resulting heat flux (Chantrenne \& Barrat, 2004a, 2004b, Termentzidis et al, 2009). The thermal conductivity is then obtained as the ratio of the heat flux and the temperature gradient. An alternative, but equivalent way consists in inducing a heat flux and to measure the resulting temperature gradient (Muller-Plathe, 1997). In both cases the system is first allowed to reach a steady state, after which prolong simulations are conducted allowing to obtain correct statistical measurements (Stevens et al, 2007). The NEMD method is often the method of choice for studies of nanomaterials (Poetzsch \& Botter, 1994) while for bulk thermal conductivity, particularly of high-conductivity materials, equilibrium method is typically preferred due to less severe size effects. The size effects in the determination of the thermal conductivity using NEMD can be understood using the following simple line of thought (Schelling \& al 2002). Imagine a phonon travelling in the crystal between the two heat reservoirs. This phonon can experience at least two kinds of scattering events: either it can be scattered by other phonons travelling in the material, or it can be scattered by the reservoirs which are seen by the considered phonon as a different material with an almost infinite thermal conductivity. The mean free path associated with this former mechanism can be roughly approximated by $\Lambda_{\text {reservoir }}=L / 2$, where $L$ is the distance between the reservoirs, because on average a phonon will travel ballistically a distance L/ 2 before being scattered by the heat source/sink. Now invoking Mathiessen rule to predict the effect of reservoir scattering on the overall thermal conductivity, the effective mean free path is given by:

$$
1 / \Lambda(L)=2 / L+1 / \Lambda(L \rightarrow \infty)
$$

where the last term $\Lambda(L \rightarrow \infty)$ describes the phonon-phonon interaction in a bulklike medium. The length dependence of the thermal conductivity can now be estimated using kinetic theory of gas: $\lambda(L)=c v \Lambda(L) / 3$ where $c$ is the heat capacity per unit volume, $v$ is the mean group velocity. Of course, this analysis is simplified because we have assumed that the effect of phonon-phonon scattering can be described in terms of a 
single mean free path, and we should have rather done the analysis mode by mode (Sellan et al 2010).

However, the previous simple kinetic model predicts:

$$
1 / \lambda(L)=1 / \lambda(L \rightarrow \infty)+6 /(c v L)
$$

and the size dependence is found to be consistent with MD NEMD simulations (Schelling et al 2002, Termentzidis et al 2009). Because of the algebraic decay, size effects in NEMD are severe, and sometimes it is faster to run equilibirum simulations to determine the thermal conductivity.

We now illustrate the determination of the thermal conductivity using the direct method with the exemple of superlattices composed of a regular alternative arrangement of solid layers, having different physical properties. Thermal transport in superlattices is characterized by the cross-plane and the in-plane conductivities, which correspond respectively to heat flowing in the direction perpendicular or parallel to the interfaces.

The geometry used for the determination of the cross-plane and in-plane thermal conductivities using NEMD is given in figure 3.1.1. Periodic boundary conditions are used in all directions, while in all cases the heat flux is imposed in z-direction. The interatomic interactions are described with Lennard-Jones (LJ) potentials, with energy unit $\varepsilon_{\mathrm{ij}}=1.0$ and length unit $\sigma_{\mathrm{ij}}=1.0$. The use of LJ potential is justified by the interest in focusing on the main phenomena introduced by the interface roughness of the super-lattices (for all the following results). The two types of materials $\mathrm{A}$ and $\mathrm{B}$ may represent respectively the $\mathrm{Si}$ and $\mathrm{Ge}$, if one considers $\mathrm{Si} / \mathrm{Ge}$-type superlattice (paragraph 5) or the GaAs and AlAs in case of GaAs/AlAs-type superlattice (paragraph 6). The two solids have the same lattice constants and they differ only by the mass ratio of the atoms constituting the superlattices layers. For the Si/Ge-type superlattices the mass ratio is taken to be 2.0, while for GaAs/AlAs this ratio is equal to 1.5. This mass ratio is consistent with the acoustic impedance ratio in the case of $\mathrm{Si} / \mathrm{Ge}$ (1.78) and in the case of GaAs/AlAs (1.2), as the ratio of acoustic impedances is equal to the square root of the ratio of masses (Swartz \& Pohl, 1989).

In this chapter the equilibrium (EMD) and the non-equilibrium (NEMD) methods are presented and the results of the thermal conductivity are reported. For both methods the molecular dynamics code LAMMPS is used (Plimpton, 1995, 1997).

In the NEMD method or direct method, a temperature gradient is imposed across the structure under study. Depending on the boundary conditions the geometry of the thermostats can change. For periodic boundary conditions there is one thermostat (cold or hot) at the middle of the slab and a second thermostat (hot or cold) is divided in two parts, which are positioned at the two edges of the slab (fig. 1). This configuration gives the opportunity to increase the statistics (double the results). The thermal conductivity is then calculated using the Fourier's law monitoring the thermal power exchange and the temperature profile of the system (fig. 2). The infinitive system size thermal conductivity then can be extrapolated by plotting the inverse of the thermal conductivity as a function of the inverse of the system size (Schelling et al, 2002). 


\section{Cross-plane TC}

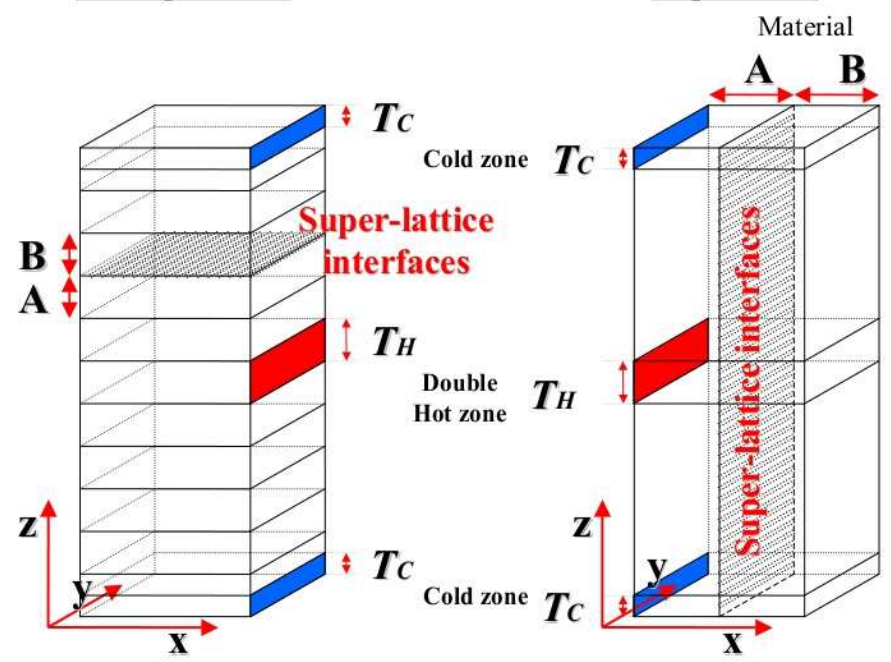

Fig. 1. Geometric configuration for the determination of the cross-plane (left) and in-plane (right) thermal conductivity simulations with NEMD and periodic boundary conditions are used in all directions.

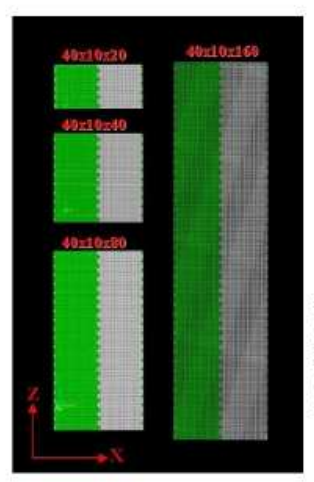

(a)

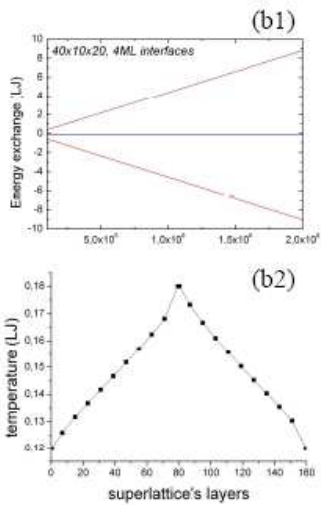

(b)

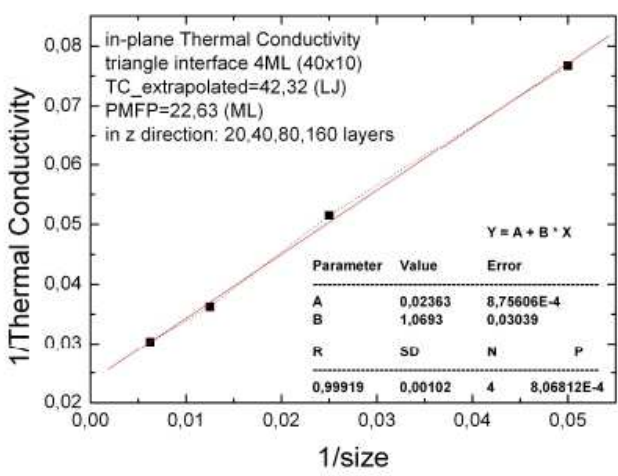

(c)

Fig. 2. To calculate the thermal conductivity (in the example the in-plane TC) of the superlattices the energy exchange (b1) and the temperature profile (b2) is extracted for each of the structures (at least 4 structures with increasing length see (a)). Then the inverse thermal conductivity is plotted as a function of the inverse of the size of slab (c). The last diagram helps in calculating the thermal conductivity of an infinitive size superlattice. 


\subsection{Equilibrium molecular dynamics method (EMD)}

Alternately, the thermal conductivity of a model system can be determined using equilibrium simulations (Shelling et al, 2002). The principle relies on the fact that the regression of the thermal fluctuations of an internal variable, in our case the thermal flux, obeys macroscopic laws. Hence, the time decay of the fluctuations of the flux is proportional to the thermal conductivity. This is mathematically expressed by the Green-Kubo formulae which state that the time integral of the heat flux autocorrelation function is proportional to the thermal conductivity tensor (Evans \& Morris, 1990):

$$
\lambda_{\alpha, \beta}=\frac{1}{V k_{B} T^{2}} \int_{0}^{+\infty}\left\langle J_{\alpha}(t) J_{\beta}(0)\right\rangle d t
$$

where $V$ is the volume of the system, and $J_{\alpha}$ denotes the component of the heat flux vector along the direction $\alpha$. The equilibrium method consists then in computing the corresponding autocorrelation, which requires following the dynamics of a system over time scales a few times larger than the longest relaxation time present in the system. In the case of heat transfer in solid materials, the longest relaxation times correspond to long wavelengths phonons (a few $\mathrm{nm}$ ) with a lifetime on the order of 100 picoseconds.

The advantage of the equilibrium method is that it allows to compute the full conductivity tensor from one simulation, which may be appreciated in superlattice simulations for instance, which display large thermal anisotropies. Another advantage of the equilibrium method is that it does not suffer from severe finite size effects as NEMD, mainly because in EMD the phonons are not strongly scattered by the boundaries of the simulation box. We can estimate the finite size effects in EMD using the following assumptions: the thermal conductivity measured in a MD simulation is given by:

$$
\lambda=\int_{\omega_{c}(L)}^{\omega_{D}} g(\omega) c(\omega) v^{2}(\omega) \tau(\omega) d \omega
$$

where

$$
\omega_{c}(L)=\omega(k=\pi / L)
$$

is the low pulsation cut-off introduced by the periodic boundary conditions, and the upper bound is

$$
\omega_{D}=\omega(k=\pi / a)
$$

where $a$ is the interatomic step, since high frequency phonons are supposed to contribute weakly to the overall thermal conductivity. In the latter expression of the thermal conductivity, $c(\omega)=k_{B}$ is the heat capacity which obeys Dulong and Petit law, $v(\omega)$ is the phonon group velocity, and $\tau(\omega)$ is the relaxation time of phonon having a pulsation $\omega$. In the following, we have ignored the possible different polarization states of the phonons to simplify the discussion. The error in the determination of the thermal conductivity is thus: 


$$
\delta \lambda=\int_{0}^{\omega_{c}(L)} g(\omega) c(\omega) v^{2}(\omega) \tau(\omega) d \omega
$$

and if we assume that for sufficient low wavevectors that the medium behaves like a Debye solid, $g(\omega) \sim \omega^{2}$, the group velocity is constant and the phonon relaxation time is supposed to obey Callaway model: $\tau(\omega) \sim \omega^{-2}$, then the error in the conductivity scales as $\delta \lambda \sim \omega_{c}(L) \sim a / L$ and thus decreases faster as the error in NEMD $\delta \lambda_{N E M D} \sim \Lambda(L \rightarrow \infty) / L$.

This allows obtaining good estimates of the thermal conductivity with rather small systems. The main drawback is intrinsic to the method, as we need to probe small thermal fluctuations around equilibrium over long time scales, which requires also performing several statistical averages over different initial conditions.

\subsection{Thermal boundary resistance}

Apart from the thermal conductivity, both NEMD and EMD simulation techniques may be used to calculate the thermal boundary resistance characterizing heat transport across the interface between two media (Barrat, 2003). The thermal boundary resistance (TBR), also known as the Kapitza resistance is defined as the ratio of the temperature jump at the interface $\Delta T$ over the heat flux $J$ crossing the interface:

$$
R_{K}=\frac{\Delta T}{J}
$$

The Kapitza resistance is thus a measure of the ability of phonons to be transmitted by the interface, with common values falling in the range between 0,01 and $0,1 M W^{-1} \cdot m \cdot K$ (Cahill et al 2003) depending on the dissimilarity between the two media. There have been several models to predict the TBR between two solids (Swartz \& Pohl 1987, 1989). Among the most popular, let us mention the acoustic mismatch model (AMM) (Khalatnikov 1952) in which the transmission of phonons is assumed to be specular and depends only the acoustic impedance mismatch between the two media, in a way similar to Snell Descartes's law ruling the transmission of an electromagnetic wave across the interface between two dielectrics with different optical indexes. The other popular model is the so-called diffuse mismatch model (DMM) (Swartz \& Pohl 1989), in which it is assumed that the phonons are diffusively scattered by the interface, and the transmission coefficient depends in this limit in the mismatch of the density of states characterizing the two media. Generally speaking, both models fail to predict the thermal boundary resistance of real interfaces (Cahill et al 2003), and a deep physical understanding of interfacial heat transport between two solids is still missing. In this context, MD may be a convenient tool to probe the transmission of phonons across ideal surfaces, and also has the flexibility to introduce defects at the interface and study their effect on interfacial heat transport. Again, two routes may be followed to calculate the interfacial resistance: in NEMD simulations, the interface to be charaterized is placed between two heat reservoirs fixed at a distance a few atomic layers away from the interface. A net heat flux in the direction perpendicular to the interface is created, and the resulting temperature jump at the interface is measured, allowing to derive the interfacial resistance. With NEMD method only the TBR of smooth interfaces can be 
calculated, as for rough interfaces there are geometrical issues about the crosssection/effective surface of the interfaces.

Equilibrium simulations rely again on the Green-Kubo formula for the interfacial conductance G=1/R (Puech 1986):

$$
G=\frac{1}{A k_{B} T^{2}} \int_{0}^{+\infty}\langle q(t) q(0)\rangle d t
$$

where $\mathrm{A}$ is the interfacial area and $\mathrm{q}(\mathrm{t})$ is the instantaneous value of the flux flowing across the interface.

The latter quantity may be computed in a MD simulation using the power of interfacial forces (Barrat 2003):

$$
q(t)=\sum_{i \in 1, j \in 2} \vec{F}_{i j} \cdot \vec{v}_{i}
$$

where the indexes 1 and 2 denote the two media separating the interface to be studied, and we have assumed pair potentials to simplify. Equilibrium simulations have been performed for interface between simple Lennard Jones solids, where the dissimilarity between the two solids is introduced by changing the acoustic impedance ratio between the two solids. The results were found to disagree with NEMD determinations of the Kapitza conductance (McGaughey 2006), especially for solids with a weak acoustic mismatch. These discrepancies are not surprising and can be traced back to the formulation of the equations themselves (Pettersson 1990), as the Green-Kubo formula above predicts a finite conductance for the interface between two identical media, while of course in NEMD one measures an infinite conductance in this situation. Maybe for this reason, most of the MD works on Kapitza resistance have considered the direct method.

Among significant work, let us mention Stevens et al. 2007, who showed that the DMM and AMM models underpredicts the thermal boundary conductance obtained by NEMD, which is found to increase with the temperature, in contrast with the theoretical models predicting a constant value, at least above the Debye temperature of the softer solid. The authors showed also that the existence of a large lattice mismatch induces interfacial stress, which deteriorates thermal transport significantly. The existence of grain boundaries has been shown also to increase the interfacial resistance (Schelling 2004).

Further work is needed, in particular using equilibrium molecular dynamics to compare MD with the available theoretical models.

\section{Heat transport in nanostructured materials}

Thermal transport in nanostructured materials has attracted an increasing international interest in the last decades. From a theoretical point of view, nanostructured materials are platforms for testing novel phonon and electron transport theories. From the research and development point of view, nanomaterials are promising candidates for nanoscale on chip coolers (Zhang \& Li, 2010). A high density of interfaces, which is the source of phonon scattering, appeared in advanced technological devices affecting their reliability and performance. Phonon interactions are modified significantly in nanostructures due to the 
dimensional confinement of the phonon modes. This effect shares some similarities with the electron confinement in a quantum well (Stroscio \& Dutta 2001). Phonon engineering in nanostructures can be succeeded in tailoring the phonon modes through the designing of the dimensions and the roughness of nanostructured materials.

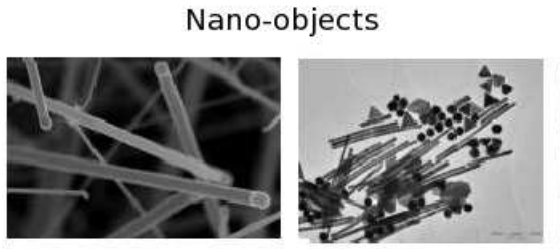

Si nanowires

Au nanoobjects
Nano-structured Materials

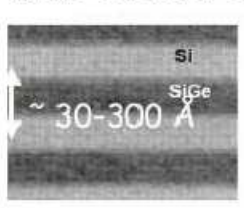

Superlattices

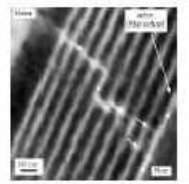

Nanosequence interfaces
Nano-systems

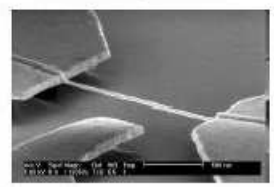

NEMS

Fig. 3. Several types of nanostructured materials, including nano-wires, nano-objects, superlattices etc.

Nanomaterials are composite materials with at least one characteristic dimension smaller than $0.5 \mu \mathrm{m}$. Super-lattices, nanofilms, nanowires, nanotubes and nanoparticles are typical examples of such materials (fig. 3). Nanomaterials exhibit transport properties different from their constituents and a fundamental understanding of heat conduction at the nanoscale is absolutely necessary to tailor their properties. Heat conduction on nanoscale differs significantly from heat transfer laws describing macroscopic transport. Among others, nanoscale energy transport can be controlled by phonon confinement (Montroll, 1950), the modification of the density of states induced by the confinement and also the ballistic behaviour of phonons. At low temperatures, phonons may travel ballistically in the bulk of the nanostructured material being scattered only by the interfaces, the latter mechanism providing the scattering mechanism which controls the thermal conductivity of the material. The crucial parameter describing ballistic transport is the ratio of the phonon mean free path (MFP) over the characteristic lengths of the nanostructure (eg. for 3Dstructures: period and roughness of the interfaces of superlattices, for 2D: interfacial roughness in case of thin films or 1D: length of nanowires). Three different regimes may be distinguished, depending on the ratio of the characteristic length of the nanostructures, over the mean free path of the energy carriers. When the characteristic length is larger than the MFP of carriers the transport is diffusive and can be described by Fourier law, while when it is smaller, the heat carriers feel the nanostructured material as a homogeneous medium with a low thermal conductivity. There is an intermediate regime, where the transport becomes ballistic and diffusion scattering becomes predominant. As we will see, this third regime offers the possibility to tailor the thermal properties of nanostructures.

Nanoscale heat transfer has indeed an old history, which can be traced back to the FermiPasta-Ulam study of heat transport in a 1D anharmonic chain (Dhar 2008). It is theoretically predicted that the thermal conductivity of model 1D chain assumptotically increases with the chain length $\mathrm{L} \lambda \propto L^{\alpha}$, at least as soon as phonon scattering is ensured by momentum conserving process (Dhar 2008). The possibility of a diverging conductivity together with thte fantastic developement of nanotechnologies in the nineties motivated experimental investigation of energy transfer in 1D systems. There has been however considerably fewer 
thermal measurement in low dimensional systems compared to electrical measurements for instance, because of the difficulty to measure directly the thermal current. Carbon nanotubes is a good paradigm of $1 \mathrm{D}$ objects, because all the heat carriers travel in the axial direction. The first experiments probing energy transfer in isolated multi-wall carbon nanotubes (Kim 2001) reported values of the thermal conductivity $\lambda \approx 3000 \mathrm{~W} / \mathrm{mK}$ even larger than the conductivity of diamond at room temperature. These large values have been confirmed by other experiments on isolated single wall (Yu 2005, Pop 2006).

This experimental effort has been accompanied by several molecular dynamics works (Berber 2000, Maruyama 2002, Zhang 2005, Yao 2005, Donadio 2007), which have concluded that the phonon mean free path may be $\mu \mathrm{m}$ and that the thermal conductivity converges for very long nanotubes (Mingo 2005, Donadio 2007). We note besides that Green-Kubo equilibrium simulations are preferred because in the direct method, it is hard to compute an effective conductivity in a material where heat is transported ballistically. Also the coupling between the nanotube and heat reservoirs in NEMD would certainly affect the conductivity measurement.

Thermal transport in nanowires has been also extensively studied during the last decade. The motivations of the first experimental works was to measure the quantum of conductance (Schwab 2000):

$$
g_{0}=\pi^{2} k_{B}^{2} T /(3 \mathrm{~h})
$$

at very low temperatures. During the years 2000, the thermal conductivity of Si nanowires at room temperatures has been measured ( $\mathrm{Li} 2003$ ) reporting values two orders of magnitude smaller than bulk Si. These low values of the thermal conductivity may be understood because in a nanowire the free surfaces induce diffuse scattering of the phonons, contrary to carbon nanotubes where phonons can only travel in the axial direction. As a consequence, the transport properties of semi-conductor nanowires depend on the state of the nanowire surface, and in particular its roughness, opening the way to achieve materials combining low thermal conductivity but electrical transport properties comparable to bulk semiconductor, with promising applications in thermoelectric conversion (Hochbaum 2008).

On the computational side, the first MD simulation of heat transport in a nanowire has been performed by Volz and Chen (Volz 1999), who already measured a two orders of magnitude reduction of the conductivity compared to the bulk. Recent simulations have confirmed the reduction, but yielding contradicting results for very small nanowires diameters (Ponomareva 2007, Donadio 2009, Abs-Da-Cruz 2011). Molecular simulations have been also pointed out the role of surface disorder on the conductivity reduction (Donadio 2009). Comparatively, there has been fewer studies on heat transport in molecular junctions, probably because of the difficulty to measure a thermal current flowing across a molecule. Molecular junctions have been however proposed to be good candidates for thermal rectifiers (Chang 2006, Casati 2007). Whang et al. recently used ultrafast thermal to probe ballistic heat transport in alkane-thiol chains supported on a gold substrate (Whang 2007). This opens the way to measure the conductance of a molecular chain as a function of its length. Theoretical studies have first considered simple one-dimensional chains (Dhar 2008, Segal 2003). Realistic models of molecular junctions have been studied recently (Mingo 2006) using Green function technique. There is relatively few molecular dynamics studies in the 
field, with the exception of the modeling of self-assembled monolayers (Luo 2010), Henry and Chen used equilibrium simulations to show that the conductivity of polyethylene chains may be orders of magnitude larger than bulk polyethylene (Henry 2008). A recent study concluded that the conductance of molecular chain is also strongly affected by its environment (Merabia 2011), with a transition between ballistic and Fourier regime.

Finally, heat transfer in the vicinity of nano-particles has also shown an increasing interest during the last years. This interest has been motivated first by the early measurements of large thermal conductivity in the so-called nanofluids, i.e. suspensions of nano-particles in a liquid solvent (Keblinski 2008). Molecular simulations have helped in solving the controversy and showed that for well dispersed nano-particles, no enhancement is expected with respect to the effective medium theory (Vladkov 2006a). These simulations have also helped in determining the interfacial thermal resistance characterising the nanoparticle/liquid interface (Vladkov 2006a). Heat transfer around strongly heated nanoparticles has also attracted attention after the development of ultrafast optical techniques which allow to selectively heat nanoparticles in suspension (Plech 2004). When the metallic particles are excited at wavelengths close to the maximum of their optical extinction, their temperature can be raised by hundreds of Kelvin, while the liquid environment in the immediate vicinity may remain at ambient temperature, thus creating very large temperature gradients and energy fluxes flowing from the nanoparticles. This raises interesting new questions regarding nanoscale heat transfer, e.g. regarding the validity of Fourier law at very large temperature gradients (Merabia 2009b), and the competition between heat transfer and boiling of the fluid surrounding the nanoparticles (Merabia 2009b). Apart from the academic interest, these questions have important biomedical applications in hyperthermia, as the appearance of vapor bubbles with submicronic radius would concentrate large thermomechanical stresses which may destroy tumors for instance. Further work is needed to understand the conditions of formation of these "nanobubbles". Let us conclude saying that nanoparticles may also served as thermal contacts to measure the conductance of molecular chains as demonstrated using MD (Merabia2011).

\section{Thermal conductivity predictions for Si/Ge superlattices, impact of rough interfaces}

Heat transport in superlattices, which are materials composed of a periodic or a random arrangement of different alternating materials with a submicronic thickness, has attracted a large scientific interest, as they exhibit low thermal conductivity (Cahill et al, 2003), at least in one direction, usually the direction normal to the interfaces. This makes superlattices promising materials for applications in MEMS and NEMS devices such as semiconductor lasers (Sale, 1995), optical data-storage media (Kim et al, 2000), thermoelectric (Hicks et al, 1993; Lin-Chung \& Reinecke, 1993) and thermomechanic devices (Ezzahri et al, 2008). For the latter two categories the thermal-conductivity characteristics are very important to ensure the correct function of the device(Daly et al, 2002). Depending on the use, the highest possible thermal conductivity is required for example to remove the Joule heat in electronic devices, or very low thermal conductivity for thermoelectric applications (Mahan 2004). With a clever tailoring of the properties of superlattice, one can succeed in both directions.

The phonon thermal conductivity of superlattices has been started to be in the center of scientific interest quite early as superlattice are promising structures for new electronic devices (Ren \& Dow 1982). It has been reported that the thermal conductivity of 
superlattices may be dramatically smaller than the corresponding values of the constituent materials in their bulk form. This decrease has been related to the folding of the Brillouin zone and the related mini-umklapp three-phonon scattering process. Tamura \& al 1999 analysed the effect on the phonon spectra in superlattices by three major reasons: a. folding of the phonon branches caused by the periodicity of the superlattices, $b$. the formation of the mini-band and c. the confinement of the acoustic phonons in the different layers due to the mismatch of the spectra. These three reasons impose reduction of the group velocity in the cross-plane direction, leading to the decrease of the cross-plane thermal conductivity. Chen \& Yang 2005a claimed that the group velocity reduction is not sufficient to explain the dramatic decrease of the thermal conductivity, and they argued that one should add the diffusive scattering at the interfaces and treat phonons as incoherent particles. Chen \& Neagu 1997 solving the Boltzmann Transport Equation for specular and diffuse interfaces showed that depending on the superlattice period, the thermal conductivity might be influenced either by the diffuse interface scattering or by the scattering induced by the dislocations. The literature is rich in this subject and a series of articles appeared with a lot of experimental (Capinski et al 1999, Huxtable et al 2002, Lee et al 1997) or theoretical results using lattice dynamics method or Equilibrium (Volz 2000, Landry 2008, Termentzidis 2011b, Termentzidis 2011c) and Non-Equilibrium Molecular Dynamics method (Liang \& Shi 2000, Chen 2004, Termentzidis 2009, Termentzidis 2010).

One very interesting property of superlattices is their thermal anisotropy. In the paragraphs below the distinction between the in-plane (parallel to the interfaces) and cross-plane (perpendicular to the interfaces) thermal conductivity has been underlined. In general one expects a value of the in-plane thermal conductivity close to the bulk conductivity especially for superlattices with smooth interfaces, where phonons are expected to specularly reflect from the interfaces, and in which each layer behaves like a phonon waveguide. For the cross-plane direction the picture is totally different, with thermal conductivity even smaller than a random alloy of the same material (Mahan 2004). A key point for the physical explanation of the phenomena related to the superlattice thermal conductivity is the thermal boundary resistance or the Kapitza resistance, which has been discussed before.

Molecular dynamics simulations have been performed recently to understand the physical mechanisms ruling the transport properties of superlattices (Landry et al, 2008, 2009, Termentzidis et al, 2009,2010,2011a,2011b,2011c). In this contribution, we will discuss the influence of the interface roughness and of the superlattice period on the in-plane and the cross-plane thermal conductivities of $\mathrm{Si} / \mathrm{Ge}$ superlattices using both the EMD and NEMD methods. This study proves that heat transport in superlattices is controlled by the interfaces. An atomic knowledge (or description) of the interfaces is necessary for the correct prediction of the thermal conductivity. In turn, understanding the link between the interfacial structure and the thermal conductivity will certainly help in tailoring and controlling the phonon behaviour in nanostructures. This can lead to the augmentation of the lifetime and to optimize the working of several nano-devices. The state of the interfaces is crucial for the determination of the behavior of phonons within the nanostructures. When the layer thickness of the superlattice is comparable with the MFP, the thermal conductivity is controlled by the transmission of phonons across the interfaces of the superlattice. In particular, the thermal boundary resistance or the Kapitza resistance, which has been discussed before, will play a key role in the thermal transport in superlattices with thin layers. 


\subsection{Modelling $\mathrm{Si} / \mathrm{Ge}$ superlattices with rough interfaces}

In this subsection, we study the effect of the superlattice period and the structure of the interface on both the in-plane and the cross-plane superlattice thermal conductivities. Simulations have been held for two periods of superlattices 20 and $40 \alpha_{0}$, which are comparable to the phonon meat free path at the temperature we are working at. To understand the role of the interfacial structure, we have considered two types of interfaces: smooth interfaces on the one hand, and rough interfaces with a right-isoscele-triangles shape, as shown in fig. 4 . In the case of rough interfaces, the height of the interfaces has been varied between 1 monolayer (ML) which is half of a lattice constant for a fcc crystal, up to $12 \alpha_{0}$, which is more than the half of the superlattice period for superlattices with period of $20 \alpha_{0}$ and from $1 \mathrm{ML}$ up to $24 \alpha_{0}$ for superlattices with period of $40 \alpha_{0}$ (see fig. 4). The maximal interface height is thus more than half the superlattice's period.

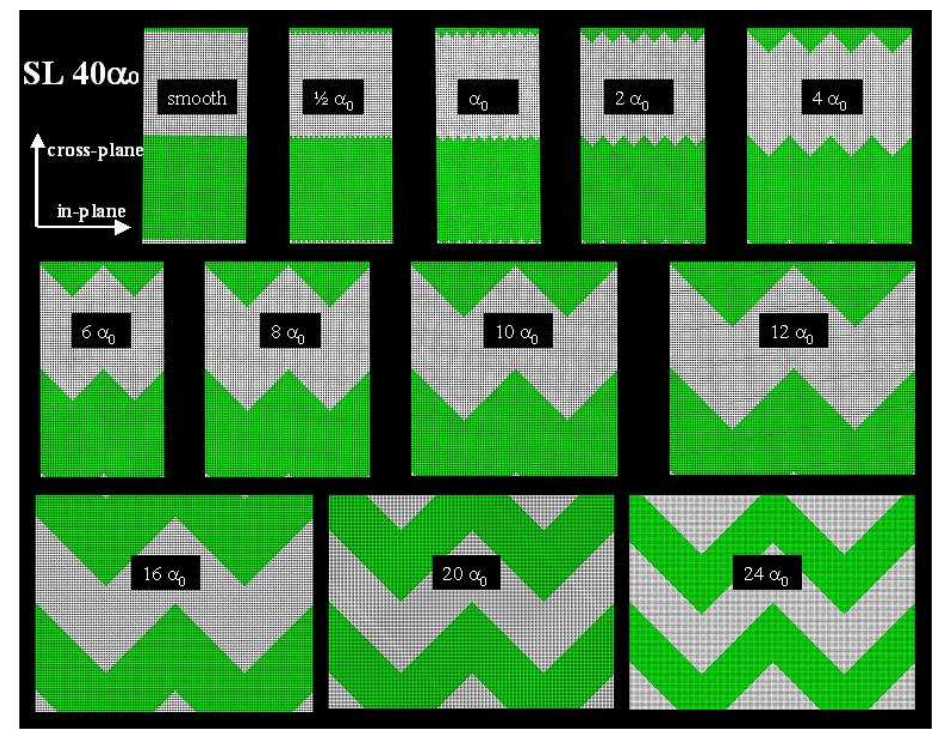

Fig. 4. Several triangular shaped interfaces with varing height, for superlattice period of $40 \alpha_{0}$.

We examined also the effect of the shape of the interface on the cross-plane and the in-plane thermal conductivities. In this case, the superlattice period is kept constant and equal to $20 \alpha_{0}$ and we considered only one height of interfaces, the $6 \alpha_{0}$ or 12MLs. Fig. 5 shows the smooth interfaces, the periodic triangular isosceles interfaces and the additional 4 other shapes. The structure shown in 5.iii is obtained by superposing the periodic isosceles of small lengths with the periodic triangular interfaces shown in 5.ii. Cosine like, random like and square like interfaces are also examined.

\subsection{Thermal conductivity of Si/Ge like superlattices with rough interfaces}

The in-plane and cross-plane thermal conductivities have been calculated using the EMD and the NEMD methods. The results are displayed in figure 6 as a function of the interface 


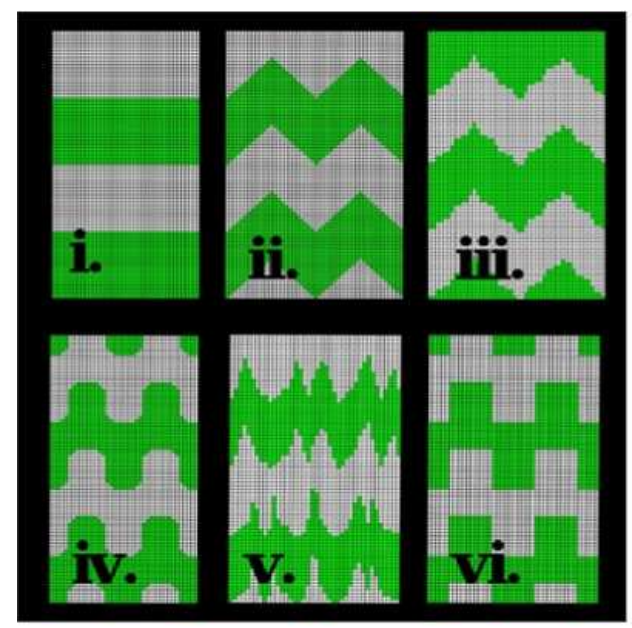

Fig. 5. Shapes of different superlattice interfaces i) smooth interfaces, ii) periodic isosceles triangles, iii) superposition of small triangles over the interfaces of the type ii, iv) cosine like v) random like, vi) periodic square like interfaces. In all cases, the height of the rough interfaces is kept constant equal to 12ML (taken from Termentzidis et al 2011b).

roughness. Again, two superlattice periods have been considered $20 \alpha_{0}$ (left) and $40 \alpha_{0}$ (right). The figures show also some points named "intra-plane" thermal conductivity, which is defined as the thermal conductivity in the direction of $45^{\circ}$ both of the in-plane or cross-plane directions. For the case of infinite roughness (with isosceles periodic triangles) the thermal conductivity in both the in-plane and the cross-plane directions is expected to be equal to this of the intra-plane conductivity, which is verified in our simulations.

For smooth interfaces, it is expected that the transmission of phonons across the interface is specular and depends only on the acoustic impedance mismatch between the bulk materials of the superlattice (Swartz and Pohl, 1987, 1989). This is actually not the case since it is observed that the thermal conductivity of the layer decreases when the film thickness decreases (at least enough to be of the same order of magnitude as the phonon mean-free path). For rough surfaces with small roughness the transmission of phonons becomes more diffusive and the transmitted phonons are distributed over a wide range of angles, which induces an additional resistance to in-plane transport. This is consistent with the MD results which conclude to a decrease of the in-plane conductivity with the interfacial roughness. For rough surfaces with large roughness there is a combination of specular and diffusive transmission. This last case shows some similarities with the smooth surface case but now specular reflection is accompanied by back scattering.

This back-scattering explains the existence of a minimum in the thermal conductivity observed for free surfaces, as well as for the in-plane conductivity of superlattices. A further increase in the surface roughness leads to higher thermal conductivity. This further increase in the thermal conductivity is related to the fact that on average, phonons experience less reflections on the asperities of the superlattice, and the mean free path length between scattering events increases, leading to an enhanced in plane transport. For the randomlike roughness, the monotonous decrease in the thermal conductivity in 
increasing the roughness's height can be interpreted if we assume that the phonon scattering at the interface remains diffusive, phenomena of back scattering and specular reflection, playing a secondary role here. Hence, we conclude that the variation in the ratio of interface roughness to the superlattice period can tailor the thermal properties of superlattices.
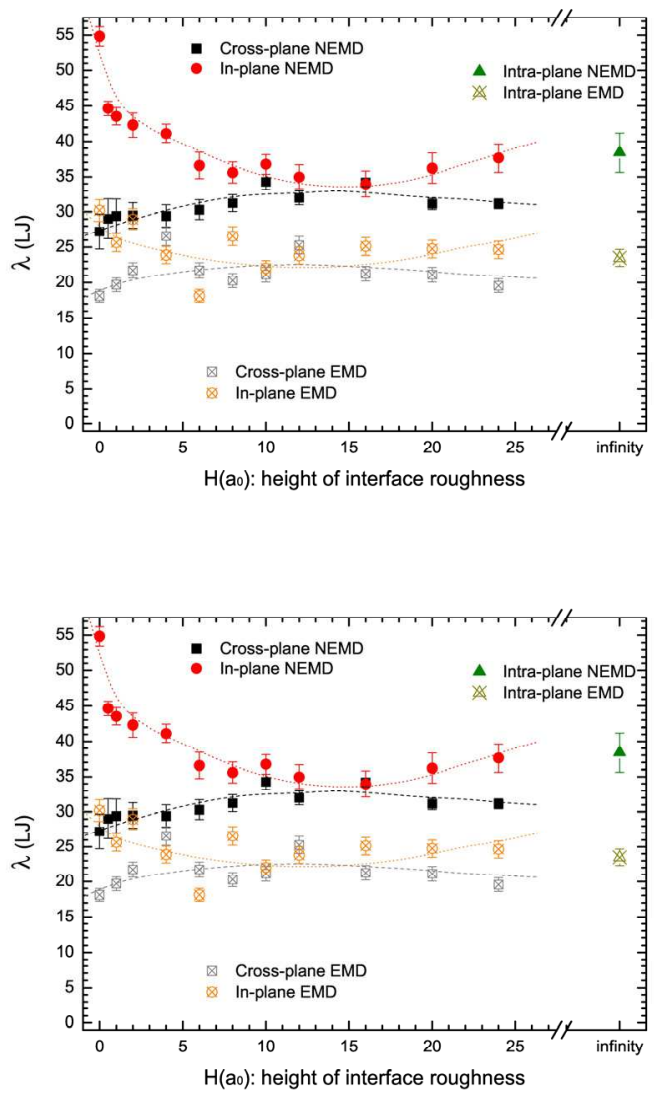

Fig. 6. In-plane and cross-plane thermal conductivity as a function of the interface roughness obtained by EMD and NEMD for superlattice with period $20 \alpha_{0}$ (top) and with $40 \alpha_{0}$ (bottom).

The cross-plane and in-plane thermal conductivities obtained by NEMD and EMD for superlattices with various shapes of interfaces are plotted in figure 7. Further details about the modelling of these interfaces and the physical explanation of the results are given in Termentzidis et al, 2011b. It is striking to note that for rough interfaces, the anisotropy of the thermal conductivity is drastically reduced. Regarding the anisotropy between the in-plane and cross-plane directions, we can categorize the interfaces in three different groups, first 
the smooth interfaces which displays the maximum anisotropy, with the in-plane thermal conductivity being more than twice larger than the cross-plane thermal conductivity; A second group contains totally random interfaces for which the two thermal conductivities exhibit their minimum values and a third group with periodic rough interfaces of a specific shape (triangular, square or cosine) where the thermal anisotropy is negligible compared to the uncertainties of the methods.

\section{Thermal conductivity predictions for GaAs/AIAs superlattices, impact of rough interfaces}

The GaAs/AlAs superlattices are important materials mainly for their optical properties. Simulations of the thermal conductivity of superlattices as a function of their period exhibit a minimum for period around 8 to 10 monolayers (Daly et al 2002, Imamura et al 2003, Chen 2005b), but this minimum is not observed in experimental measurements (Capinski et al 1999). The quality of the interfaces might be the reason to explain this discrepancy. For perfectly smooth interfaces the minimum exists, while for rough interfaces with a height as small as one monolayer the minimum disappears and the thermal conductivity increases with the periodicity of the superlattice. The works of Daly et al 2002 and Imamura and al 2003 are based on rough interfaces of one atomic layer and with a stochastic distribution of the two types of atoms that compose the superlattice.

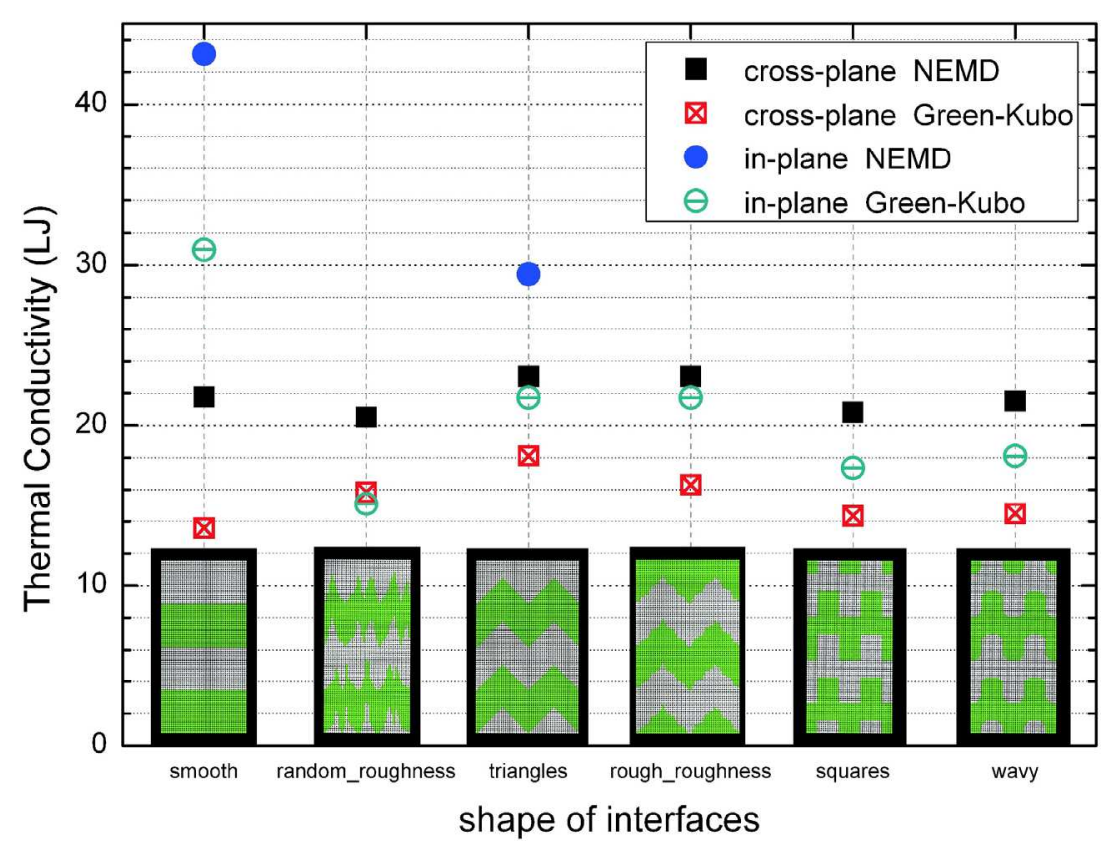

Fig. 7. In-plane and cross-plane thermal conductivities for several shapes of the interfaces obtained by EMD and NEMD for superlattices with period $20 \mathrm{a}_{0}$ and with constant height of interfaces $6 \mathrm{a}_{0}$. 


\subsection{Modelling rough interfaces for the GaAs/AIAs superlattices}

A new modeling of realistic interfaces is considered with the present study (Termentzidis et al 2010, 2011a). Interfaces with square formed islands of one monolayers and pyramide like islands of two monolayers are modelled. Furthermore there are two characteristic lengths of square islands depending if GaAs or AlAs is on top. It has been proven that large scale islands are formed when an AlAs layer grows on a GaAs layer while small scale islands are formed when a GaAs layer grows on an AlAs layer (Tanaka \& Sakaki 1987, Jusserand et al 1990). Furthermore interfaces with interdiffusion parts are also considered. Figure 8 depicts the two kind of interfaces with interface height of one monolayer and in figure 9 with two monolayers (these figures are taken from Termentzidis et al 2010)

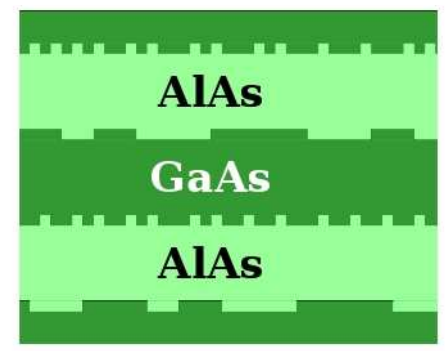

Fig. 8. Schematic representation of the two different scale interfaces between the GaAs/AlAs and the $\mathrm{AlAs} / \mathrm{GaAs}$, for height of interfaces of one monolayer - square like interfaces.
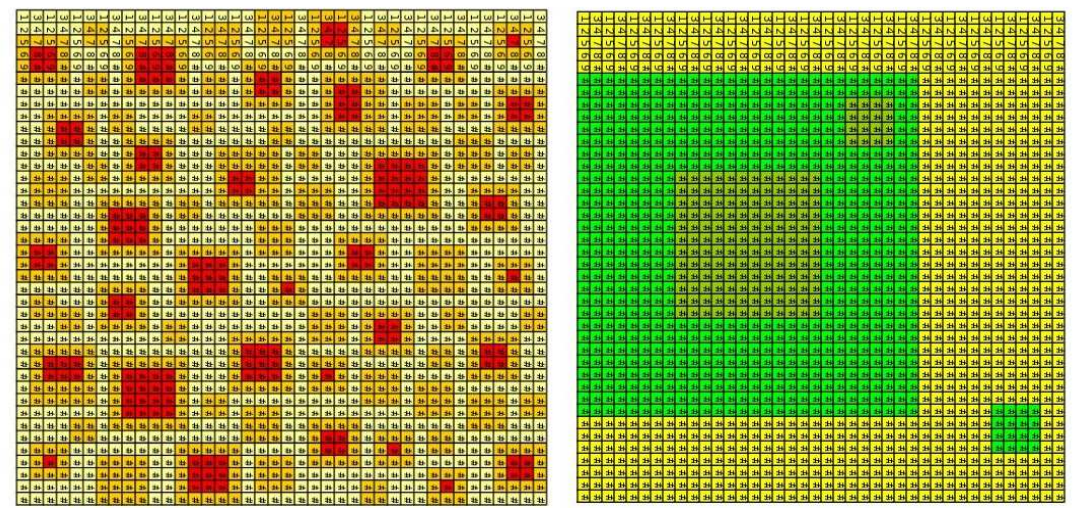

Fig. 9. Schematic representation of the two different scale interfaces between the GaAs/AlAs and the AlAs/GaAs, for height of interfaces of two monolayers - pyramoidal like interfaces (taken from Termentzidis et al 2010).

\subsection{Thermal conductivity of GaAs/AIAs like superlattices with rough interfaces}

Figure 10 shows the predicted cross-plane thermal conductivity as a function of the superlattice period, for a variety of interface configurations. In figure 10 at left the thermal conductivity is plotted for smooth interfaces, for rough interfaces with height of one monolayer and three coverage factors $(1 \%, 10 \%$ and $50 \%)$ and finally for interfaces with 
height of 2 monolayers. The coverage factor of $1 \%$ exhibits the minimum in thermal conductivity as the smooth interfaces. For coverage factor of $10 \%$ and $50 \%$ the minimum dissapears and the results confirm previous theoretical observations (Daly et al 2002, Imamura et al 2003), while the two defect concentrations do not influence the thermal conductivity. For height of roughness of $2 \mathrm{MLs}$, the thermal conductivity is higher than for smooth interfaces and exhibits a maximum for a $8 \alpha_{0}$. In the same figure at the right the influence of interdiffusion of the two species is shown, with similar behavior as the interfaces of height of two MLs. These unexpected results are related with the fact that inelastic scattering could enhance the thermal conductivity through interfaces (Lepi et al 2003). The study shows that the thermal conductivity depends strongly on the detailed description of the interfaces, including height, shape of roughness, interdiffusion of species etc. Changing the structure of the interface can favor or deteriorate the thermal transport through interfaces, and we showed that the interface structure is a relevant parameter which controls the thermal properties of superlattices.
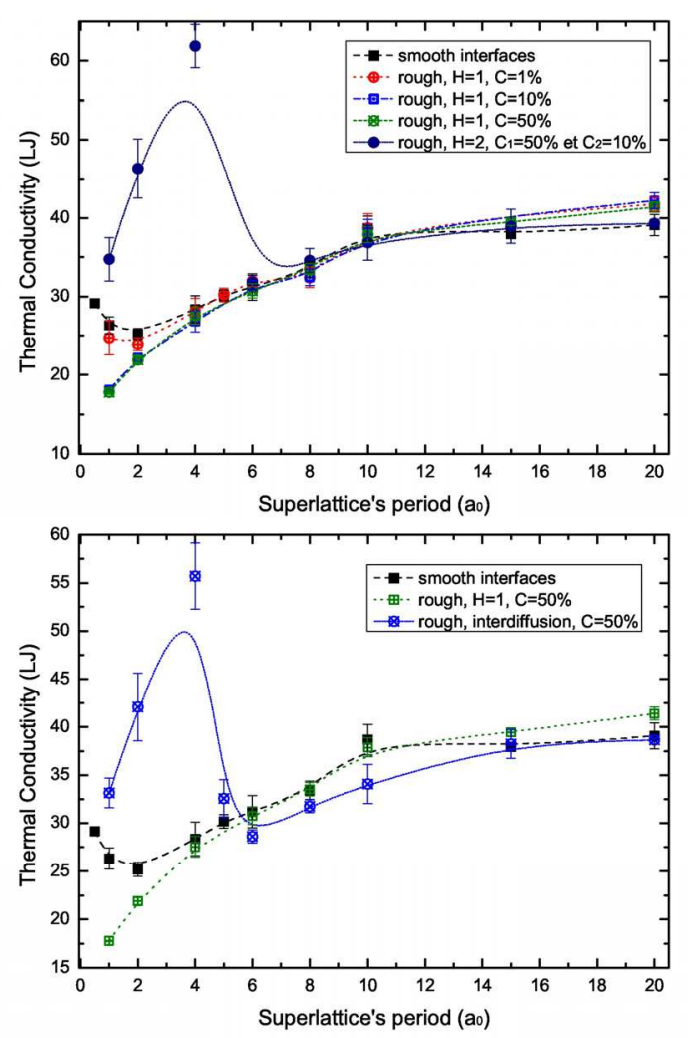

Fig. 10. Cross-plane thermal conductivity as a function of the superlattice period with NEMD for GaAs/AlAs systems. Left: smooth interfaces, rough interfaces of height of 1ML with three different concentrations and rough interfaces of height of $2 \mathrm{ML}$ are presented. Right: rough interfaces with interdiffusion. The smooth and rough interfaces with a concentration of $50 \%$ are also shown for comparison. 


\section{Conclusion}

We hope we have helped in showing the possibilities of the molecular dynamics technique to probe heat transport in solids, and in particular nanomaterials. Molecular dynamics is a relatively simple and flexible method to be used especially today when stable optimized open source codes have become avalaible: LAMMPS, DLPOLY, GROMACS to name a few. The ever increasing number of publications has helped in resolving controversies regarding heat transfer at the nanoscale, and also getting physical insights in classical problems. A physical understanding of energy transport across two solids-a very simple question-still poses a challenge ! MD simulations may help in observing the scattering of phonons at interfaces, which certainly complements experimental investigations. In the context of nanomaterials, MD is well adapted to characterize ballistic heat transport in nano-objects, although care should be taken not to introduce spurious sources of scattering ! Let us hope that further experimental measurements may improve the modeling of the nanomaterials that we have considered.

\section{References}

Abs-Da-Cruz, C. Termentzidis, K. Chantrenne, P. \& Kleber, X. (2011). Molecular Dynamics simulations for the prediction of thermal conductivity of bulk silicon and silicon nanowires: Influence of interatimic potentials and boundary condictions. Journal of Applied Physics, Vol 110, No. 3, (August 2011), pp 034309, doi:10.1063/1.3615826

Alder, BJ and Wainwright, TE. (1957). Phase Transition for Hard Sphere System. Journal of Chemical Physics, Vol 27, No. 5, (August 1957), pp 1208-1209, doi:10.1063/1.1743957

Alder, BJ and Wainwright, TE. (1959). Studies in Molecular Dynamics. I. General Method. Journal of Chemical Physics, Vol 31, No. 2, (Febrouary 1959), pp 459-466, doi:10.1063/1.1730376

Allain MP. \& Tildesley DJ. (1987). Computer Similution of Liquids. Oxford University Press, ISBN: 0-19 8556454 (Pbk), New York, USA

Barrat, JL. \& Chiaruttini, F. (2003). Kapitza resistance at the liquid-solid interface. Molecular Physics, Vol 101, No. 11, pp 1605-1610, doi:10.1080/0026897031000068578

Berber, S. Kwon, YK. \& Tomanek, D. (2000). Unusual High Thermal Conductivity of Carbon Nanotubes. Physical Review Letters, Vol. 84, pp 4613-4617,

DOI:10.1103/PhysRevLett.84.4613

Cahill, DG. et al. (2003). Nanoscale thermal transport. J. Applied Physics, Vol 93, No. 2 (August 2002), pp 793-818, DOI:10.1063/1.1524305

Callaway J. (1959) Model for Lattice Thermal Conductivity at Low Temperatures, Physical Review, Vol 113, pp 1046-1051, DOI:10.1103/PhysRev.113.1046

Capinski, W. Maris, H. Ruf, T. Cardona, M. Ploog, K. and Katzer D. (1999). Thermalconductivity measurements of GaAs/AlAs superlattices using a picosecond optical pump-and-probe technique. Phys. Rev. B, Vol 59, No. 12 (March 1999), pp 81058113, DOI:10.1103/PhysRevB.59.8105

Casati, G. (2007), Device Physics: The Heat on is off. Nature Nanotechnology, Vol 2, pp 2324, DOI: 10.1038/nnano.2006.191

Chang, CW. Okawa, D. Majumdar, A. and Zettl, A. (2006). Solid-state Thermal Rectifier. Science. Vol 314, pp 1121 -1124, DOI: 10.1126/ science.1132898 
Chantrenne, P \& Barrat, LJ. (2004a). Analytical model for the thermal conductivity of nanostructures. Superlatt. Microstruct. , Vol 35, pp 173-186

Chantrenne, P \& Barrat, LJ. (2004b). Finite size effects in determination of thermal conductivities: comparing molecular dynamics results with simple models. J. Heat Transfer, Vol 126, pp 577-585

Chantrenne, P. (2007). Molecular Dynamics, In: Microscale and Nanoscale Heat Transfer, Sebastian Volz, pp. (155-179), Springer-Verlag Berlin and Heidelberg GmbH \& Co.K, ISBN-10: 3540360565, Berlin, Germany

Chen, G. \& Neagu, M. (1997). Thermal Conductivity and Heat Transfer in Superlattices. Applied Physics Letters, Vol 71, pp 2761-2763, DOI:10.1063/1.120126

Chen, G. \& Yang, RG. (2005a). Nanostructured Thermoelectric Materials : from Superlattices to Nanocomposites. Material Integration, special issue, Vol 18

Chen, Y. Li, D. Lukes, JR. Ni Z. \& Chen, M. (2005b). Minimum superlattice thermal conductivity from molecular dynamics. Physical Review B, Vol. 72 (Novembre 2005), pp 174302, DOI:10.1103/PhysRevB.72.174302

Chen, Y. Li, D. Yang, J. Wu, Y. Lukes, JR. \& Majumdar, A. (2004). Molecular Dynamics study of the lattice thermal conductivity of $\mathrm{Kr} / \mathrm{Ar}$ superlattice nanowires. Physica B : Condensed Matter, Vol 349, No. 1-4 (June 2004), pp 270-280,

DOI:10.1016/j.physb.2004.03.247

Daly, BC. Maris, HJ. Imamura, K. \& Tamura, S. (2002). Molecular Dynamics calculation of the thermal conductivity of superlattices. Physical Review B, Vol 66, No. 2 (June 2002), pp 024301, DOI:10.1103/PhysRevB.66.024301

Dammak H., Chalopin Y., Laroche M., Hayoun M. And Greffet J-J. (2009) Quantum Thermal Bath for Molecular Dynamics Simulation, Physical Review Letters, Vol. 103, pp 190601 - 190604 DOI:10.1103/PhysRevLett.103.190601

Daw, MS and Baskes MI (1984) Embedded-atom method: derivation and application to impurities, surfaces and other defects in metals, Phys. Rev. B, Vol 29, 6443-6453 DOI 10,1103/PhysRevB,29,6443

Dhar, A. (2008). Heat Transport in Low-dimensional systems. Advances in Physics, Vol 75, No. 5, (Dec 2008), pp 457-537

Donadio, D. \& Galli, G. (2007). Thermal Conductivity of Isolated and Interacting Carbon Nanotubes : Comparing Results from Molecular Dynamics and the Boltzmann Transport Equation. Physical Review Letters, Vol. 99, pp 255502,

DOI:10.1103/PhysRevLett.99.255502

Donadio, D. \& Galli, G. (2009). Atomistic Simulations of Heat Transport in Silicon Nanowires. Physical Review Letters, Vol. 102, pp 195901, DOI: 10.1103/PhysRevLett.102.195901

Dove M.T. (2005) Introduction to Lattice Dynamics, Cambridge University Press, ISBN 0521398940, Cambridge United Kingdom

Evans, DJ. (1982). Homogeneous NEMD algorithm for thermal conductivity-Application of non-canonical linear response theory. Physics Letters A, Vol 91, No. 9, (October 1982), pp 457-460, doi:10.1016/0375-9601(82)90748-4

Evans, DJ. (1986). Thermal Conductivity of the Lennrd-Jones fluid. Physical Review A, Vol 34, No. 2, (August 1986), pp 1449-1453, doi:10.1103/PhysRevA.34.1449

Evans, DJ. \& Morris, GP. (1990). Statistical Mechanics of nonequilibrium liquids, Cambridge University Press, ISBN-13 978-0-521-85731-8, New York 
Ezzahri, Y. Zeng, G. Fukutani, K. Bian, Z. \& Reinecke, TL. (2008). A comparison of thin film microfrigerators based on Si/SiGe superlattice and bulk SiGe. Microelectronics J., Vol 39, (August 2007), pp 981-991, DOI:10.1016/j.mejo.2007.06.007

Frenkel, D \& Smit, B. (1996). Understanding Molecular Simulation: From Algorithms to Applications, Academic Press, ISBN: 0-12-267351-4, San Diego

Hansen, JP. \& Klein, ML. (1976). Dynamical Structure Factor of Rare-Gas Solids. Physical Review B. Vol 13, pp 878-887, DOI: 10.1103/PhysRevB.13.878

Henry, A. \& Chen, G. (2008). High Thermal Conductivity of Single Polyethylene Chains Using Molecular Dynamics Simulations. Physical Review Letters, Vol. 101, pp 235502, DOI: 10.1103/PhysRevLett.101.235502

Hicks, LD. Harman, TC. \& Dresselhaus, MS. (1993). Use of quantum-well superlattices to obtain a high figure of merit from nonconventional thermoelectric materials. Applied Physics Letters, Vol 63, No. 23 (October 1993), pp 3230-3232, DOI:10.1063/1.110207

Hochbaum, AI. Chen, RK. Delgado, RD. Liang, WJ. Garnett, EC. Najarian, M. Majumdar, A. and Yang, PD. (2008). Nature, Vol. 451, pp 163-U5, DOI: 10.1038/nature06381

Holland, MG. (1963) Analysis of Lattice Thermal Conductivity, Physical Review 132, pp 2461-2471, DOI: 10.1103/PhysRev.132.2461

Huxtable, S. et al (2002). Thermal conductivity of Si/SiGe and SiGe/SiGe superlattices. Applied Physics Letters, Vol 80, No. 10 (March 2002), pp 1737-1739,

DOI:10.1063/1.1455693

Imamura, K. Tanaka, Y. Nishiguchi, S. Tamura, S. and Maris, HJ. (2003). Lattice thermal conductivity in superlattices: molecular dynamics calculations with a heat reservoir method. J. Physics: Condensed Matter, Vol 15, No. 50 (December 2003), pp 86798690, DOI:10.1088/0953-8984/15/50/002

Jusserand, B. Mollot, F. Moisson, JM. And Le-Roux, G. (1990). Atomistic-scale roughness of GaAs/AlAs interfaces: A Raman scattering study of asymmetrical short-period superlattices. Applied Physics Letters, Vol 57, No. 6 (May 1990), pp 560-562, DOI:10.1063/1.103646

Keblinski, P. Prasher, R. \& Eapen, J. (2008). Thermal Conductance of Nanofluids: Is the Controversy Over? J. Nanoparticle Res., Vol. 10, p 1089-1097

Khalatnikov, IM. (1952). Zh. Eksp. Teor. Fiz. 22, pp 687.

Kim, E. et al. (2000). Thermal boundary resistance at Ge2Sb2Te5/ZnS:SiO2 interface. Applied Physics Letters, Vol 76, No. 26 (April 2000), pp 3864-3866, DOI:10.1063/1.126852

Kim, P. Shi, L. Amjumdar, A. \& McEuen, PL. (2001) Thermal Transport Measurements of Individual Multiwall Nanotubes. Physical Review Letters. Vol. 87, pp 215502215505, DOI:10.1103/PhysRevLett.87.215502

Kotake, S \& Wakuri S. (1994). Molecular dynamics study of heat conduction in solid materials. JSME International Journal, Series B: Fluids and Thermal Engineering, Vol 37, No. 1, (Febrouary 1994), pp 103-108, ISSN:09148817

Ladd, AJC., Moran, B. and Hoover, WG. (1986), Lattice thermal conductivity: a comparison of molecular dynamics and anharmonic lattice dynamics. Physical Review B, Vol 34, No. 8, pp 5058-5064, DOI:10.1103/PhysRevB.34.5058 
Landry, ES. Hussein, MI. \& McGaughey, AJH. (2008). Complex superlattice unit cell designs for reduced thermal conductivity. Physical Review B, Vol 77, No. 18, pp 18184302, doi:10.1103/PhysRevB.77.184302

Landry, ES. \& McGaughey, AJH. (2009). Thermal boundary resistance predictions from molecular dynamics simulations and theoretical calculations. Physical Review B, Vol 80, No. 16, pp 16165304, doi:10.1103/PhysRevB.80.165304

Lee, SM. Cahill, DG. \& Venkatasubramanian, R. (1997). Thermal conductivity of Si-Ge superlattices. Applied Physics Letters, Vol 70, (June 1997), pp 2957-2959, DOI:10.1063/1.118755

Lepri, S. Livi, R. \& Politi, A. (2003). Thermal conduction in classical low-dimensional lattices. Physics Reports, Vol 377, (October 2002), pp 1-80, DOI:10.1016/S03701573(02)00558-6

Li, D. Wu, YY. Kim, P. Shi, L. Yang, PD. \& Majumdar, A. (2003). Thermal Conductivity of Individual Silicon Nanowires. Applied Physics Letters. Vol. 83, pp 2934-2936. DOI: $10.1063 / 1.1616981$

Liang, XG. \& Shi, B. (2000). Two-dimensional molecular dynamics simulation of the thermal conductance of superlaticces. Materials Science and Engineering: A, Vol 292, (Novembre 2002), pp 198-202, DOI:10.1016/S0921.5093(00)01012-1

Lin-Chung, PJ. \& Reinecke, TL. (1995). Thermoelectric figure of merit of composite superlattice systems. Physical Review B, Vol 51, No. 19 (May 1995), pp 13244-13248, DOI:10.1103/PhysRevB.51.13244

Luo, T. \& Lloyd, JR. (2010). International J. Heat Mass Transfer, Vol. 53, pp 1-11, DOI: 10.1016/j.ijheatmasstransfer.2009.10.033

Mahajan, S, Subbarayan, G \& Sammakia, BG. (2007). Estimating thermal conductivity of amorphous silica nanoparticles and nanowires using molecular dynamics simulations. Physical Review E, Vol 76, No. 5, (Novembre 2007), pp 056701, DOI:10.1103/PhysRevE.76.056701

Mahan, GD. (2004). Thermal Conductivity of Superlattices, In: Thermal Conductivity: Theory, Properties and Applications. Terry T. Tritt, pp. (153-165), Klumer Academic/Plenum Publishers, ISBN: 0-306-48327-0, New York, USA

Maruyama, S. (2002). A Molecular Dynamics Simulation of Heat Conduction in Finite Length SWNTs. Physica B, Vol. 323, pp 193-195, DOI 10.1016/S0921-4526(02) 00898-0

McGaughey, AJH. \& Kaviany, M. (2004) Quantitative validation of the Boltzmann transport equation phonon thermal conductivity model under the signle-mode relaxation time approximation. Physical Review B. Vol. 69, pp 094303-1, 094303-12, DOI:10.1103/PhysRevB.69.094303

Mcgaughey, AJH. \& Li, J. (2006) Molecular Dynamics Prediction of the Thermal Resistance of Solid-Solid Interfaces in Superlattices, Proceedings of IMECE 2006, ASME International Mechanical Engineering Conference and Exhibition, November 5-10, 2006, Chicago USA

Merabia, S. Shenogin, S. Joly, L. Keblinski, P. \& Barrat, JL. (2009a). Proc. Nat. Ac. Sci., Vol. 106, pp 15113-15118, DOI: 10.1073/pnas.0901372106

Merabia, S. Keblinski, P. Joly, L. Lewis, LJ. \& Barrat, JL. (2009b). Critical Heat Flux Around Strongly Heated Nanoparticles. Physical Review E, Vol. 79, pp 021404, DOI: 10.1103/PhysRevE.79.021404 
Merabia, S. Barrat, JL. \& Lewis, L. (2011). Heat Conduction across Molecular Junctions Between Nanoparticles. J. Chemical Physics. Vol. 134. pp 234707, DOI: 10.1063/1.3600667

Mingo, N. \& Broido, DA. (2005). Carbon Nanotube Ballistic Thermal Conductance and Its Limits. Physical Review Letters. Vol. 95, pp 096105, DOI: 10.1103/PhysRevLett.95.096105

Mingo, N. (2006). Anharmonic phonon flow through molecular-sized junctions. Physical Review B. Vol. 74, pp 125402, DOI: 10.1103/PhysRevB.74.125402

Montroll, E. (1950). Size Effect in Low Temperature Heat Capacities. J. Chemical Physics, Vol 18, No. 2, (August 1949), pp 183-185, DOI:10.1063/1.1747584

Mûller-Plathe, F. (1997). A simple nonequilibrium molecular dynamics method for calculating the thermal conductivity. J. Chemical Physics, Vol 106, No. 14, (January 1997), pp 6082-6085, DOI:10.1063/1.473271

Pettersson, S. \& Mahan, GD. (1990) Theory of the Thermal Boundary Resistance between dissimilar lattices, Physical Review B, Vol. 42, No. 12, pp 7386-7390, DOI:10.1103/PhysRevB.42.7386

Plech, A. Kotaidis, V. Grésillon, S. Dahmen, C. \& Von-Plessen, G. (2004) Laser-induced heating and melting of gold nanoparticles studied by time-resolved X-ray scattering. Physical Review B. Vol. 70, pp 195423

Plimpton, S. (1995). Fast Parallel Algorithms for Short-Range Molecular Dynamics. J. Computational Physics, Vol 117, (Mars 1995), pp 1-19.

Plimpton, S. Pollock, R. and Stevens, M. (1997). Particle-Mesh Ewald and rRESPA for Parallel Molecular Dynamics Simulations. In Proceedings of the Eigth SIAM Conference on Parallel Processing for Scientific Computing, Minneapolis, MN, March 1997

Poetzsch, RH \& Bôttger, H. (1994). Interplay of disorder and anharmonicity in heat conduction: Molecular dynamics study. Physical Review B, Vol 50, No. 21, (December 1994), pp 15757-15763, DOI:10.1103/Phys.RevB.50.15757

Ponomareva, I. Srivastava, D. \& Menon, M. (2007). Thermal Conductivity in thin Silicon Nanowires: Phonon Confinement Effect. NanoLetters. Vol. 7. pp 1155-1159

Pop, E. Mann, D. Wang, Q. Goodson, K. \& Dai, H. (2006). Thermal Conductance of an Individual Single-wall carbon nanotube above room Temperature. NanoLetters. Vol 6, pp 96-100, DOI: 10.1021/nl052145f

Puech, L. Bonfait, G. and Castaing, B. (1986) Mobility of the He-3 Solid Liquid InterfaceExperience and Theory Journal of Low Temperature Physics, Vol 62, pp 315-327, DOI: $10.1007 /$ BF00683467

Rahman, A. (1964). Correlations in the Motion of Atoms in Liquid Argon. Physical Review, Vol 136, No. 2A, (May 1964), pp A405-A411, doi:10.1103/Phys.Rev.136.A405

Rahman, A and Stillinger, F. (1974). Propagation of sound in water. A molecular-dynamics study. Physical Review A, Vol 10, No. 1, (March 1974), pp 368-378, doi:10.1103/Phys.RevA.10.368

Ren, SY. \& Dow, J. (1982). Thermal Conductivity of Superlattices. Physical Review B, Vol 25, (March 1982), pp 3750-3755, DOI:10.1103/Phys.RevB.25.3750

Sale, TE. (1995). Cavity and Reflector Design for Vertical Cavity Surface Emitting Lasers, Proceedings of SIOE'94, SCardiff, UK, March 1994 
Schelling, PK. Phillpot, SR. \& Keblinski, P. (2002). Comparison of atomic-level simulation methods for computing thermal conductivity. Physical Review B, Vol 65, No. 14, (April 2002), pp 144306, DOI:10.1103/PhysRevB.65.144306

Schelling, PK. Phillpot, SR. and Keblinski, P, (2004) Kapitza conductance and phonon scattering at grain boundaries by simulation, J. Applied Physics, Vol. 95, No 11, pp 6082-6090

Schwab, K. Henriksen, EA. Worlock, JM. \& Roukes, ML. (2000). Measurement of the Quantum of Thermal Conductance, Nature, Vol. 404, pp 974-977, ISSN: 00280836

Segal, D. Nitzan, A. Hanggi, P. (2003). Thermal Conductance through Molecular Wires, J. Chemical Physics. Vol. 119, pp 6840-6855, DOI: 10.1063/1.1603211

Stevens, RJ, Zhigilei, LV \& Norris, PM. (2007). Effects of temperature and disorder on thermal boundary conductance at solid-solid interfaces: Nonequilibrium molecular dynamics simulations. International J. Heat and Mass Transfer, Vol 50, No. 19-20, (September 2007), pp 3977-3989, DOI:10.1016/j.ijheatmasstransfer.2007.01.040

Stillinger F.H. and Weber T.A. (1985) Computer simulations in condensed phases of silicon. Physical Review B. Vol 31, pp 5262-5271 DOI 10,1103/PhysRevB,31,5262

Stroscio, M. \& Dutta, M. (2011). Phonons in nanostructures, Cambridge University Press, ISBN-0-521-79279-7, Cambridg, United Kingdom

Swartz, ET. \& Pohl, RO. (1987). Thermal resistance at interfaces. Applied Physics Letters, Vol 51, No. 26 (October 1987), pp 2200-2202, DOI:10.1063/1.988939

Swartz, ET. \& Pohl, RO. (1989). Thermal boundary resistance. Review Modern Physics, Vol 61, No. 3, pp 605-668, DOI:10.1103/RevModPhys.61.605

Tamura, S. Tanaka, Y. \& Maris, HJ. (1999). Phonon group velocity and thermal conduction in superlattices. Physical Review B, Vol 60, (July 1999), pp 2627-2630, DOI:10.1103/Phys.RevB.60.2627

Tanaka, M. \& Sakaki, H. (1987). Atomistic models of interface structures of GaAs-AlxGa1$x$ As $(x=0.2-1)$ quantum wells grown by interrupted MBE. Journal of Crystal Growth, Vol 81, No. 1-4, pp 153-158, DOI:10.1016/0022-0248(87)90383-6

Termentzidis, K, Chantrenne, P \& Keblinski, P. (2009). Nonequilibrium molecular dynamics simulations of the in-plane thermal conductivity of superlattices with rough interfaces. Physical Review B, Vol 79, No. 21, (June 2009), pp 214307,

DOI:10.1103/PhysRevB.79.214307

Termentzidis, K. Chantrenne, P. Duquense, JY. \& Saci, A. (2010). Thermal conductivity of GaAs/AlAs superlattices and the puzzle of interfaces. J. Physics: Condensed Matter, Vol 22, No. 47, (November 2010), pp 475001, DOI:10.1088/0953$8984 / 22 / 47 / 475001$

Termentzidis, K. Chantrenne, P. Duquense, JY. \& Saci, A. (2011a). Molecular dynamics calculations of the cross-plane thermal conductivity of GaAs/AlAs superlattices with rough interfaces. Chinese J. Physics, Vol 49, No. 1, (Febrouary 2011), pp 443447.

Termentzidis, K. Merabia, S. Chantrenne, P. \& Keblinski, P. (2011b). Cross-plane thermal conductivity of superlattices with rough interfaces using equilibrium and nonequilibrium molecular dynamics. International J. Heat and Mass Transfer, Vol 54, No. 9, (April 2011), pp 2014-2020, ISSN:0017-9310 
Termentzidis, K. Parasuraman, J. Abs Da Cruz, C. Merabia, S. Angelescu, D. Marty, F. Bourouina, T. Kleber, X. Chantrenne, P. and Basset (2011c). Thermal conductivity and thermal boundary resistance of nanostructures. Nanoscale Research Letters, Vol 6, No. 1, (April 2011), pp 288, DOI:10.1186/1556-276X-6-288.

Tersoff, J. (1986) New empirical model for the structural properties of silicon, Physical Review Letters, Vol 56, pp 632-635, DOI:10.1103/PhysRevLett.56.632

Tersoff, J. (1988a) New empirical approach for the structure and energy of covalent systems, Physical Review B, Vol 37, pp 6991-7000, DOI:10,1103/PhysRevB.37,6991

Tersoff, J. (1988b) Empirical interatomic potential for silicon with improved elastic properties, Physical Review B, Vol. 38, pp 9902- 9905,

DOI:10.1103/PhysRevB.38.9902

Verlet, L. (1967) Computer "Experiments" on Classical Fluids. I. Thermodynamical Properties of Lennard-Jones Moleculaes. Physical Review, Vol 159, (July 1967), pp 98-103, DOI:10,1103/PhysRev.159.98

Verlet, L. (1968) Computer "Experiments" on Classical Fluids. II. Equilibrium Correlation Functions. Physical Review, Vol 165, (January 1968), pp 201-214, DOI:10,1103/PhysRev.165.201

Vladkov, M. \& Barrat, JL. (2006a). Modeling Transient Absorption and Thermal Conductivity in a simple Nanofluid, NanoLetters, Vol. 6, pp 1224-1228, DOI: 10.1021/nl060670o

Vladkov, M. \& Barrat, JL. (2006b ). Linear and nonlinear viscoelasticity of a model unentangled polymer melt: Molecular dynamics and Rouse mode analysis. Macromolecular Theory and Simulations. Vol. 15. pp 252-262, DOI: 10.1002/mats.200500079

Volz, S. \& Chen, G. (1999). Molecular dynamics simulation of thermal conductivity of silicon nanowires. Applied Physics Letters, Vol 75, No. 14, (October 1999), pp 2056-2058, DOI:10.1063/1.124914

Volz, S. Saulnier, JB. Chen, G. \& Beauchamp, P. (2000). Computation of thermal conductivity of $\mathrm{Si} / \mathrm{Ge}$ superllatices by molecular dynamics techniques. Microelectronics J., Vol 31, No. 9-10, (October 2000), pp 815-919, DOI:10.1016/S0026-2692(00) 00064-1

Wang, Z. Carter, JA. Lagutchev, A. Koh, YK. Seong, NH. Cahill, DG. \& Dlott, DD. (2007) Ultrafast Flash Thermal Conductance of Molecular Chains Science. Vol. 317. pp 787-790, DOI: $10.1126 /$ science. 1145220

Yang, J. (2004). Theory of Thermal Conductivity, In: Thermal Conductivity, Theory, Properties, and Applications. Terry M.Trutt, pp. (1-17), Kluwer Academic/Plenum Publishers, ISBN 0-306-48327-0, New York

Yao, Z. Wang, JS. Li, B. \& Liu, GR. (2005). Thermal Conduction of Carbon Nanotubes Using Molecular Dynamics. Physical Review B. Vol. 71, pp 085417,

DOI:10.1103/PhysRevB.71.085417

Yu, C. Shi, L. Yao, Z. Li, D. \& Majumdar, A. (2005). Thermal Conductance and thermopower of an individual single-wall carbon nanotube. NanoLetters, Vol 5, pp 1842-1846, DOI: $10.1021 / \mathrm{nl} 051044 \mathrm{e}$

Zhang, G. \& Li, B. (2005). Thermal Conductivity of Nanotubes Revisited: Effects of Chirality, Isotope Impurity, Tube Length, and Temperature. J. Chemical Physics, Vol. 123, pp 114714, DOI: $10.1063 / 1.2036967$ 
104 Molecular Dynamics - Theoretical Developments and Applications in Nanotechnology and Energy

Zhang, G \& Li, B. (2010). Impacts of doping on thermal and thermoelectric properties of nanomaterials. Nanoscale, Vol 2, (May 2010), pp 1058-1068,

DOI:10.1039/c0nr00095g 


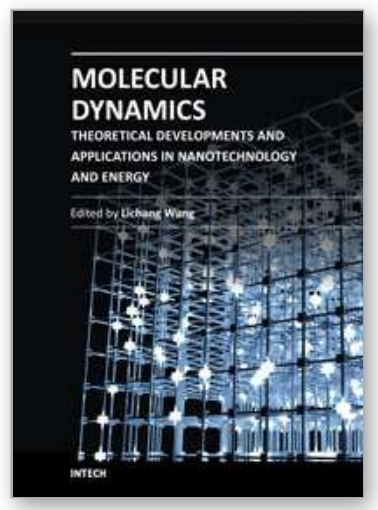

\author{
Molecular Dynamics - Theoretical Developments and Applications \\ in Nanotechnology and Energy \\ Edited by Prof. Lichang Wang
}

ISBN 978-953-51-0443-8

Hard cover, 424 pages

Publisher InTech

Published online 05, April, 2012

Published in print edition April, 2012

Molecular Dynamics is a two-volume compendium of the ever-growing applications of molecular dynamics simulations to solve a wider range of scientific and engineering challenges. The contents illustrate the rapid progress on molecular dynamics simulations in many fields of science and technology, such as nanotechnology, energy research, and biology, due to the advances of new dynamics theories and the extraordinary power of today's computers. This first book begins with a general description of underlying theories of molecular dynamics simulations and provides extensive coverage of molecular dynamics simulations in nanotechnology and energy. Coverage of this book includes: Recent advances of molecular dynamics theory Formation and evolution of nanoparticles of up to 106 atoms Diffusion and dissociation of gas and liquid molecules on silicon, metal, or metal organic frameworks Conductivity of ionic species in solid oxides Ion solvation in liquid mixtures Nuclear structures

\title{
How to reference
}

In order to correctly reference this scholarly work, feel free to copy and paste the following:

Konstantinos Termentzidis and Samy Merabia (2012). Molecular Dynamics Simulations and Thermal Transport at the Nano-Scale, Molecular Dynamics - Theoretical Developments and Applications in Nanotechnology and Energy, Prof. Lichang Wang (Ed.), ISBN: 978-953-51-0443-8, InTech, Available from: http://www.intechopen.com/books/molecular-dynamics-theoretical-developments-and-applications-innanotechnology-and-energy/molecular-dynamics-simulations-and-thermal-transport-at-the-nano-scale

\section{INTECH}

open science | open minds

\section{InTech Europe}

University Campus STeP Ri

Slavka Krautzeka 83/A

51000 Rijeka, Croatia

Phone: +385 (51) 770447

Fax: +385 (51) 686166

www.intechopen.com

\section{InTech China}

Unit 405, Office Block, Hotel Equatorial Shanghai

No.65, Yan An Road (West), Shanghai, 200040, China

中国上海市延安西路65号上海国际贵都大饭店办公楼 405 单元

Phone: +86-21-62489820

Fax: $+86-21-62489821$ 
(C) 2012 The Author(s). Licensee IntechOpen. This is an open access article distributed under the terms of the Creative Commons Attribution 3.0 License, which permits unrestricted use, distribution, and reproduction in any medium, provided the original work is properly cited. 